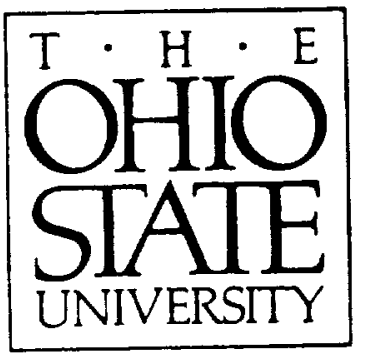

P?ク

\title{
The Helicopter Antenna Radiation Prediction Code (HARP)
}

\author{
F.T. Klevenow, B.G. Lynch, E.H. Newman, R.G. Rojas,
} J.T. Scheick, H.T. Shamansky and K.Y. Sze

\section{The Ohio State University \\ ElectroScience Laboratory \\ Department of Eloctrical Engineering \\ Columbus, Ohio 43212}

Final Report 722792-1

Grant No. NAG-1-1058

August 1990

National Aeronautics and Space Administration

Langley Research Center

Hampton, VA 23665-5225

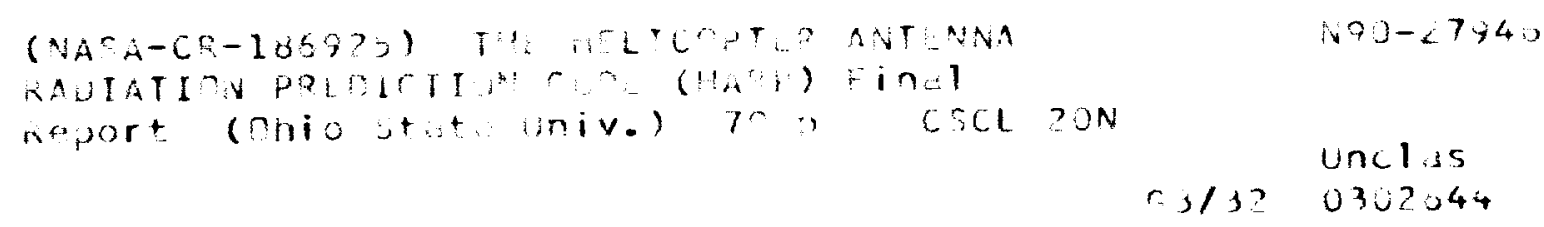




\section{NOTICES}

When Government drawings, specifications, or other data are used for any purpose other than in connection with a definitely related Government procurement operation, the United States Government thereby incurs no responsibility nor any obligation whatsoever, and the fact that the Government may have formulated, furnished, or in any way supplied the said drawings, specifications, or other data, is not to be regarded by implication or otherwise as in any manner licensing the holder or any other person or corporation, or conveying any rights or permission to manufacture, use, or sell any patented invention that may in any way be related thereto. 


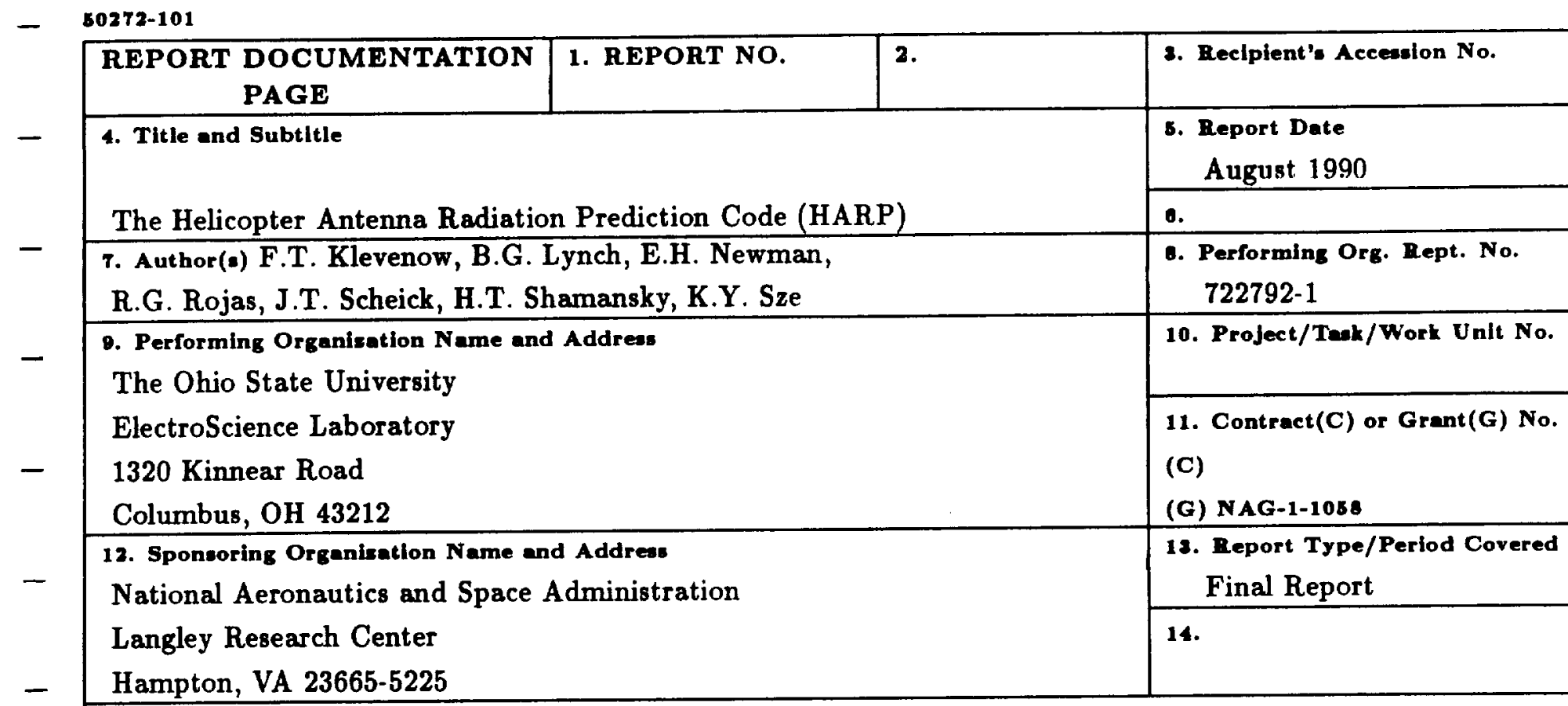

15. Supplementary Notes

16. Abstract (Limit: 200 worda)

This report will describe the first nine months effort in the development of a user oriented computer code, referred to as the HARP code, for analyzing the radiation from helicopter antennas. The HARP code uses modern computer graphics to aid in the description and display of the helicopter geometry. At low frequencies the helicopter is modeled by polygonal plates, and the method of moments is used to compute the desired patterns. At high frequencies the helicopter is modeled by a composite ellipsoid and flat plates, and computations are made using the geometrical theory of diffraction. The HARP code will provide a user friendly interface, employing modern computer graphics, to aid the user to describe the helicopter geometry, select the method of computation, construct the desired high or low frequency model, and display the results.

17. Document Analy sis a. Descriptors AIRCRAFT

GTD

3-D

ANTENNAS

MOMENT METHOD

b. Identifiers/Open-Ended Terms

c. COSATI Field/Group

18. Avallabllity Statement

A. Approved for public release;

Distribution is unlimited.

\begin{tabular}{|l|l|}
\hline $\begin{array}{l}\text { 10. Security Class (This Report) } \\
\text { Unclassified }\end{array}$ & $\begin{array}{c}\text { 21. No. of Pagea } \\
78\end{array}$ \\
\hline $\begin{array}{c}\text { 20. Security Class (This Page) } \\
\text { Unclassified }\end{array}$ & 22. Price \\
\hline
\end{tabular}




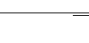




\section{Contents}

List of Figures

$\mathbf{v}$

CHAPTER

PAGE

1 Introduction

2 Overview of the Helicopter Antenna Radiation Prediction Code 3

2.1 Section 1: User Input Geometry and Spline Interpolation . 5

2.2 Section 2: Low Frequency MM Analysis . . . . . . . . . 8

2.3 Section 3: High Frequency UTD Analysis ......... 10

2.4 Section 4: Display Results .............. 12

3 Aircraft Fuselage Representation 18

3.1 Introduction . . . . . . . . . . . . . . . 18

3.2 Input Data from Blueprints ............. 18

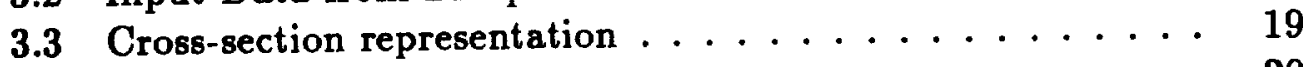

3.4 Blending along $\mathrm{y}$-axis $\ldots \ldots \ldots 20$

4 Moment Method Model Development 22

4.1 Moment Method Plate Model .............. 22

4.2 3D Graphics . . . . . . . . . . . . . . . . 24

4.3 Speeding Up the MM Analysis ... . . . . . . . . 29

5 GTD Model Development $\quad \mathbf{3 0}$

5.1 Method 1 - Four Nonlinear Equations .......... 34

5.2 Method 2 - N Linear Equations ............ 45

5.3 Method 3 - Method 1 with Penalty Function ....... 49 
$\begin{array}{lll}6 & \text { Project Status } & 54\end{array}$

\section{APPENDICES}

A High Frequency Techniques

B Low Frequency Techniques 65

C The Tektronix 4337 Workstation 70

D The Tektronix Hardware/Software Improvements 72 


\section{List of Figures}

2.1 A Block diagram of the HARP code. . . . . . . . 4

2.2 A symmetric cross section of the LHX helicopter. . . . . . 13

2.3 A spline fit is made to produce a smooth contour from the discrete data points entered by the user. . . . . . . . . 14

2.4 Several contour are combined to produce a continuous surface model of a portion of the LHX fuselage. . . . . . . 15

2.5 In the UTD model, the fuselage of the helicopter is modeled by two ellipsoids, joined at the antenna location. . . . . . 16

2.6 A ellipse is fit to a fuselage cross section. . . . . . . . . 17

3.1 A comparison of chord length and pseudo-arc length smoothing. .................... 21

4.1 A continuous surface representation of a helicopter . . . . 25

4.2 A plate model of a helicopter with arrows indicating the MM surface patch dipole modes used by AMC. . . . . . 26

5.1 A composite ellipsoid. . . . . . . . . . . . . 31

5.2 The parameters which define a shifted ellipse. . . . . . . 33

5.3 A four point fit by means of the SDA. . . . . . . . 38

5.4 A four point fit by means of the SDA. . . . . . . 39

5.5 A four point fit by means of the SDA. . . . . . . . 40

5.6 A four point fit by means of the SDA. . . . . . . . . 41

5.7 A four point fit by means of the SDA. . . . . . . . 42

5.8 A four point fit by means of the SDA. . . . . . . . 43

5.9 A five point fit by means of the LEM. . . . . . . . . . 47

5.10 A five point fit by means of the LEM. . . . . . . . . 48 
5.11 Results obtained with a five point fit for various antenna locations. .................... 49

5.12 Penalty function $P(x)\left(e_{1}=1, e_{2}=0.1\right)$ and its first and second derivatives. ............... 51

B.1 The computed (dashed line) and measured (solid line) far zone pattern of a short monopole on the underside of a helicopter computed by AMC. 


\section{Chapter 1}

\section{Introduction}

Modern helicopters have a number of antennas to accomplish a wide variety of tasks. In order to investigate the performance of an antenna mounted on an helicopter, it is common to build scale models of the helicopter and perform a great number of measurements. An alternative to this procedure is to develop analytical techniques that can be used to analyze the radiation of an antenna in the presence of a complicated structure such as an aircraft or a helicopter. Two computational techniques that complement each other have been developed to handle this problem. One technique, commonly known as the Uniform Geometrical Theory of Diffraction (UTD) $[1,2]$, is a high frequency technique which can be used to compute near and far field patterns of sources radiating in the vicinity of electrically large objects. The second technique, known as the Moment Method (MM), is applicable to objects which are not electrically large. [3]. Thus, for a given problem, depending on the electrical size of the structures, one can choose the method which is more appropriate to calculate the radiation patterns of the antennas under study. 
This report will describe the first nine months of effort in developing a computer code which combines modern computer graphics and MM and UTD computational techniques into an integrated package for analyzing the far zone radiation pattern of antennas on helicopters. The code will be referred to as the Helicopter Antenna Radiation Prediction Code (HARP), and will be run via an $\mathrm{X}$ windows menu driven user interface on a Tektronics 4337 3D Graphics Workstation. For more information on the hardware, see Appendix C. Since the MM and UTD codes are largely in place $[4,22,23]$, the main effort in developing HARP will be the development of a user friendly interface to the EM codes. This interface will employ computer graphics to allow the user to easily specify the helicopter shape and antenna location, and then obtain radiation patterns using either the MM or UTD. Although HARP is being designed specifically for helicopters, it is actually applicable to almost any geometry consisting of a main body or fuselage, of essentially arbitrary shape, with at tached wings and fins. Thus, the HARP code should be applicable to aircraft, helicopters, missiles, spacecraft, etc.

Chapter 2 of this report will present an overview of the HARP code. Techniques for obtaining smooth and accurate spline representations of the fuselage are described in Chapter 3. The MM and GTD modeling of the helicopter are presented in Chapters 4 and 5, respectively. Chapter 6 is a summary of the project status. The appendices briefly describe the MM, the GTD, and the main Tektronics hardware and software. 


\section{Chapter 2}

\section{Overview of the Helicopter Antenna Radiation Prediction Code}

This section will present an overview of the Helicopter Antenna Radiation Prediction Code (HARP). Figure 2.1 shows a block diagram of HARP. It is worth noting that of the 13 "boxes", only two (i.e., "Perform MM Computations" and "Perform UTD Computations") refer to electromagnetics. The rest refer to the input, manipulation, and display of the helicopter geometry. This is an accurate reflection of the fact that the vast majority of our effort in developing HARP deals with geometry, not electromagnetics.

The are four main parts to the flow diagram of Figure 2.1:

1. The user enters the helicopter geometry as cross sections for the fuselage and flat plates for the wings and fins. A spline interpolation of the fuselage cross sections is performed to produce a continuous surface representation of the fuselage (see Chapter 3 ). The continuous surface model is displayed on the CRT screen. 


\section{THE HELICOPTER ANTENNA RADIATION PREDICTION CODE}

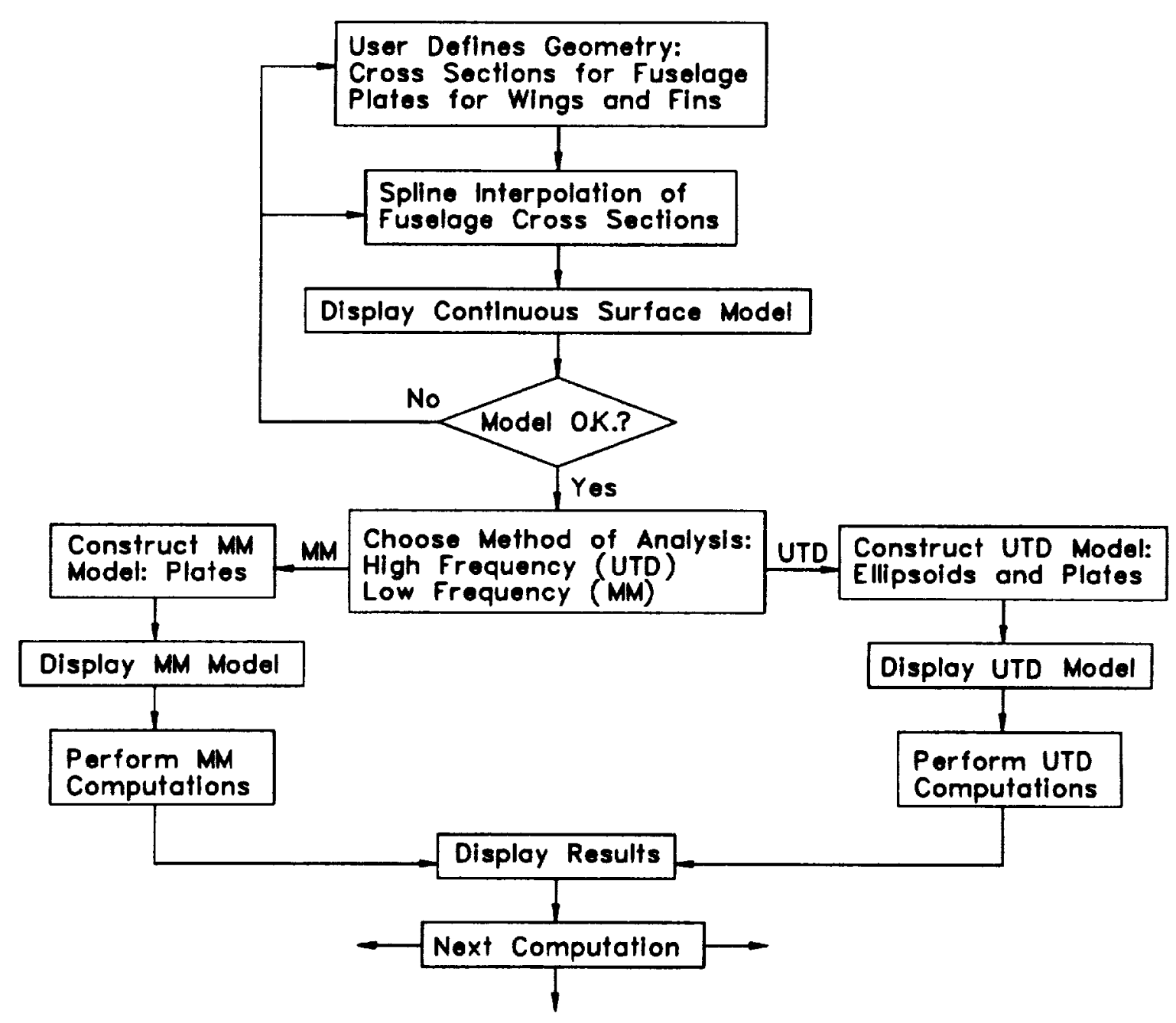

Figure 2.1: A Block diagram of the HARP code. 
2. If the helicopter is to be analyzed via the MM, a plate model is constructed and displayed on the CRT screen. The plate model is analyzed by the low frequency method of moments Aircraft Modeling Code (AMC) (see Appendix B or reference [22]).

3. If the helicopter is to be analyzed via the UTD, a composite ellipsoid and flat plate model is constructed and displayed on the CRT screen. The composite ellipsoid and flat plate model is analyzed by the high frequency UTD code NEWAIR3 (see Appendix A or reference [4]).

4. The final step is to display the antenna far zone radiation patterns.

The four sections are described in more detail below.

\subsection{Section 1: User Input Geometry and Spline Interpolation}

User Defines Geometry:

The HARP system begins with the user entering the shape to be analyzed. The fuselage is entered by defining its cross section at a number of stations from the nose to the tail end. Depending upon complexity, typically a fuselage can be adequately defined by $5-20$ cross sections. If the fuselage axis is the $y$ axis, then a cross section is defined by the $x, z$ coordinates of a discrete number of points on the cross section. Depending upon complexity, a cross section can typically be defined by $8-40$ points. Thus, depending upon complexity and desired accuracy, a user can typically expect to enter between 40 and 800 points to define a fuselage. We have developed a 
graphical aid to the user in entering this data. In brief, it is assumed that the user has a set of blueprints or engineering drawings which contain sufficient cross sections to define the fuselage. The cross sections are placed on a digitizing tablet, and the cross hairs of a puck are moved along the cross section. Periodically, the digitizing button on the puck is depressed, thus entering the coordinates of a point on that cross section. As points are entered, the cross section is displayed in real time on the CRT screen.

To illustrate this process, Figure 2.2 shows one-half of a symmetric fuselage cross section from the blueprints of the LHX helicopter. The points in Figure 2.3 illustrate the digitized sample points entered by the user to describe this cross section. The solid line shows the spline fit to the digitized points. Note that the spline fit provides a smooth and accurate representation of the true cross section of Figure 2.2, including both the convex and concave portions of the cross section. Finally, Figure 2.4 shows how several cross sections are combined to form a continuous surface model of a portion of the LHX helicopter fuselage.

After the fuselage cross sections are entered, the next step is to define the wings, fins, and other objects attached to the fuselage such as rotor blades and the radiating antenna. Wings and fins will be modeled by flat plates, while the radiating antenna will be modeled by thin wires. The next years effort will develop graphical aids and user friendly methods for entering this data.

\section{Spline Interpolation of Fuselage Cross Sections:}

As described above, the user defines the fuselage by a discrete number of cross sections, each of which is defined by a discrete number of points. 
This limited set of input data may not be sufficient or in the proper form to be used by the EM codes. Thus, the next step is to make a spline fit to the cross section data entered by the user. Spline fits to discrete data can introduce anomalies or bumps, especially near sharp corners. We have developed a modification of the periodic cubic splines which avoids these anomalies. The algebraic nature of the spline fit essentially gives us a continuous surface approximation of the fuselage shape entered by the user. Virtually any information needed concerning the fuselage can be extracted from this spline fit, and the spline fit now defines the fuselage to HARP. See Chapter 3 for more details concerning the spline fit to the fuselage.

Display Helicopter Model:

The next step is to display the helicopter model on the CRT screen. The display will be a shaded $3 \mathrm{D}$ color image of the continuous surface spline interpolation of the fuselage and of the attached wings and fins, rotor blades, antenna, etc. (see Chapter 4.2). If the user is not satisfied with the computer model of the helicopter he can return to the geometry input sections of the code and either correct errors in the original input data, or enter more cross sections or more points per cross section to improve the model. This process of alternately entering and displaying geometry continues until the user is satisfied with the model. At this point the model can be stored on the system disk for later retrieval.

Choose Method of Analysis:

Once the model is deemed correct, the next step is to choose the method of EM analysis. Basically there a two choices. For electrically small bodies one normally employs the method of moments (MM). For electrically 
large bodies one normally uses the uniform geometrical theory of diffraction (UTD). These methods are described in Appendices A and B. Initially the user will select the method of analysis based upon his own judgement. Later, HARP will select and display a default method, which will be used unless overridden by the user.

\subsection{Section 2: Low Frequency MM Analysis}

\section{Construct MM Model:}

If the $\mathrm{MM}$ is to be the method of analysis, the first step is to construct a model which is compatible with the low frequency Aircraft Modeling Code (AMC) [22]. AMC requires the fuselage to be defined by cross sections, and the wings and fins by flat plates. A major portion of the first years effort has been to convert the continuous surface or spline fit model of the fuselage into cross sections which are compatible with AMC. This is not trivial because of the need for the grid formed by the fuselage cross sections to exactly match the wing and fin attachments. In the AMC code insuring that wings and fins would properly attach to the fuselage is the responsibility of the user. In the HARP code it is done automatically.

Although the conversion from the continuous surface spline fit to the fuselage cross sections required by $\mathrm{AMC}$ is essentially automatic, the user does have some control. For example, he can specify the number of cross sections and the number of points per cross section, or he can simply take the code defaults. Note that the user initially specified the fuselage in terms of cross sections. Next a spline fit was made to the cross sections defined by 
the user. Finally, the spline fit was re-converted back to cross sections. It may at first seem that the spline fit was an unnecessary step since we start and end with fuselage cross sections. However, the spline fit is essential because the fuselage cross sections entered by the user are virtually never the same as that needed by AMC.

AMC takes the fuselage cross section data and converts it to a tiled surface or plate model of the fuselage. Since the wings and fins are also modeled by plates, the low frequency model is a $100 \%$ plate model. The MM plate model is described in more detail in Chapter 4.1.

Display MM Model:

The next step in the MM analysis is to display the plate or tiled surface model of the helicopter. The plate model will be displayed on the same screen as the continuous surface model. If the user is not satisfied with the plate model, he can return to the "Construct MM Model" block and increase or decrease the number of cross sections or the number of points per cross section. See Chapter 4.2 for more details concerning the 3D graphics.

\section{Perform MM Computations:}

Once the user is satisfied with the plate model of the helicopter, the next step is to perform the MM computations in AMC. These involve computation of the MM impedance matrix, a matrix solution for the currents, and finally computation of the desired far zone patterns. Depending upon the electrical size of the helicopter, and upon the speed of the computer, this step can take between a few minutes and several hours of CPU time. 


\subsection{Section 3: High Frequency UTD Analy- sis}

Construct UTD Model:

If the UTD method is to be used to analyze the helicopter, then the helicopter must be represented by a composite ellipsoid and flat plates. The composite ellipsoid will usually model the fuselage of the helicopter, while the flat plates will model any other structure attached to or in the vicinity of the fuselage such as wings, fins, engines, blades, etc. The composite ellipsoid, which is depicted in Figure 2.5, is obtained by joining two ellipsoids at the plane (perpendicular to the axis of the fuselage) where the antenna is located. Note that the composite ellipsoid is uniquely specified with four parameters which must be determined from the geometry of the helicopter. Thus, the first step in the UTD analysis is to develop an algorithm which will determine these four parameters for a given helicopter geometry and antenna location.

As described in Chapter 5, a number of techniques are being studied which will allow HARP to construct the ellipsoid with minimum user input. For example, one method under consideration is to first consider the cross section of the fuselage where the antenna is located and which is perpendicular to the axis of the helicopter. In this cross section, an ellipse is fit to the true cross section of the helicopter as shown in Figure 2.6. The next step is to consider a second cross-section of the helicopter fuselage which is perpendicular to the first one, but also passes along the antenna location. This procedure will allow us to determine the four parameters of 
the composite ellipsoid.

It is important to emphasize that, in contrast to the moment method technique where the model has to be accurate everywhere, the UTD model needs to be most accurate in the vicinity of the antenna. The UTD model of the helicopter fuselage can be less accurate far from the antenna location. This is true due to the fact that in the high frequency regime, radiation, diffraction, and scattering are local phenomena. This means that the radiation characteristics of an antenna mounted on a fuselage depend most strongly on the geometry of the fuselage in the vicinity of the antenna location, and much less on the geometry of the fuselage far away from the antenna.

Once the composite ellipsoid is determined, the next step will be to develop algorithms to attach the flat plates to the fuselage. Recall that the flat plates will be used to model everything that is attached to the fuselage, i.e., fins, wings, rotor, etc. This step can become very complicated because the helicopter model depends on the antenna location. There are several UTD "rules" that are helpful when one needs to decide what parts of the helicopter should be included in the model, while leaving others out. Some of these "rules" will also be implemented in the computer to minimize the amount of user intervention needed.

Display UTD Model:

The UTD model of the helicopter, consisting of a composite ellipsoid and flat plates will be displayed on the same CRT screen as the continuous surface model. If the user is not satisfied with the model, he can return to the "Construct UTD Model" block and modify the parameters of the UTD 
model. See Chapter 4.2 for more information concerning 3D graphics.

\section{Perform UTD Calculations:}

Once the user is satisfied with the UTD model, the next step is to perform the UTD pattern computations using the UTD code NEWAIR3 [4].

\subsection{Section 4: Display Results}

Display Results

The final step in the analysis is to display the desired radiation patterns on the CRT screen. These may take the form of rectangular or polar plots. Data file will also be written onto the disk for later analysis or hard copy display of the data.

\section{Next Computation}

After the display of the results the user may either terminate the session or start another run. For example, the user may:

1. Create a new helicopter geometry to be analyzed

2. Modify the existing helicopter geometry

3. Change the antenna location on the helicopter

4. Analyze the existing geometry at a different frequency

5. Analyze the existing geometry by a different method (i.e. MM or UTD)

6. Compute different radiation pattern cuts. 


$$
\bar{\varepsilon}
$$




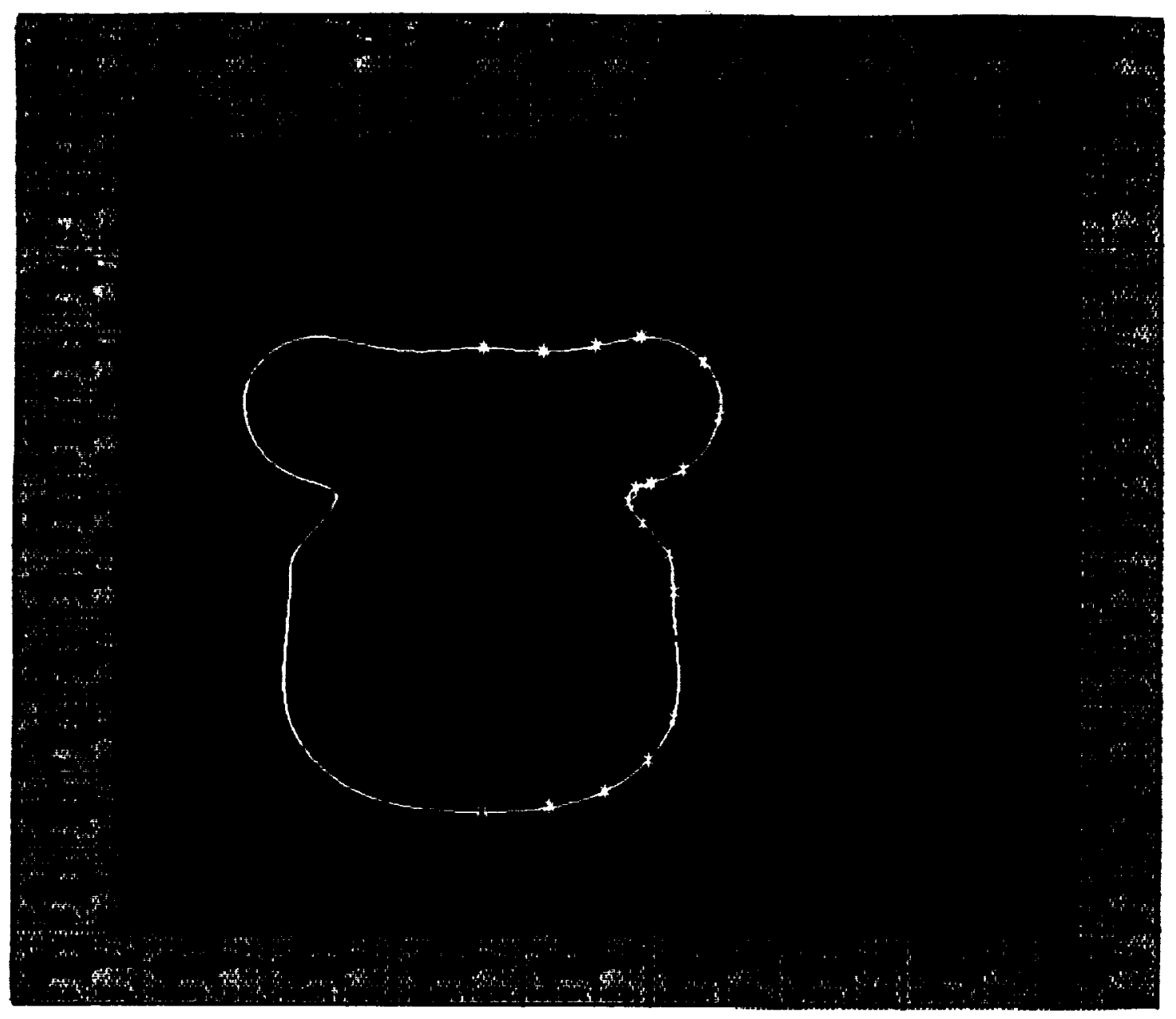

Figure 2.3: A spline fit is made to produce a smooth contour from the discrete data points entered by the user. 


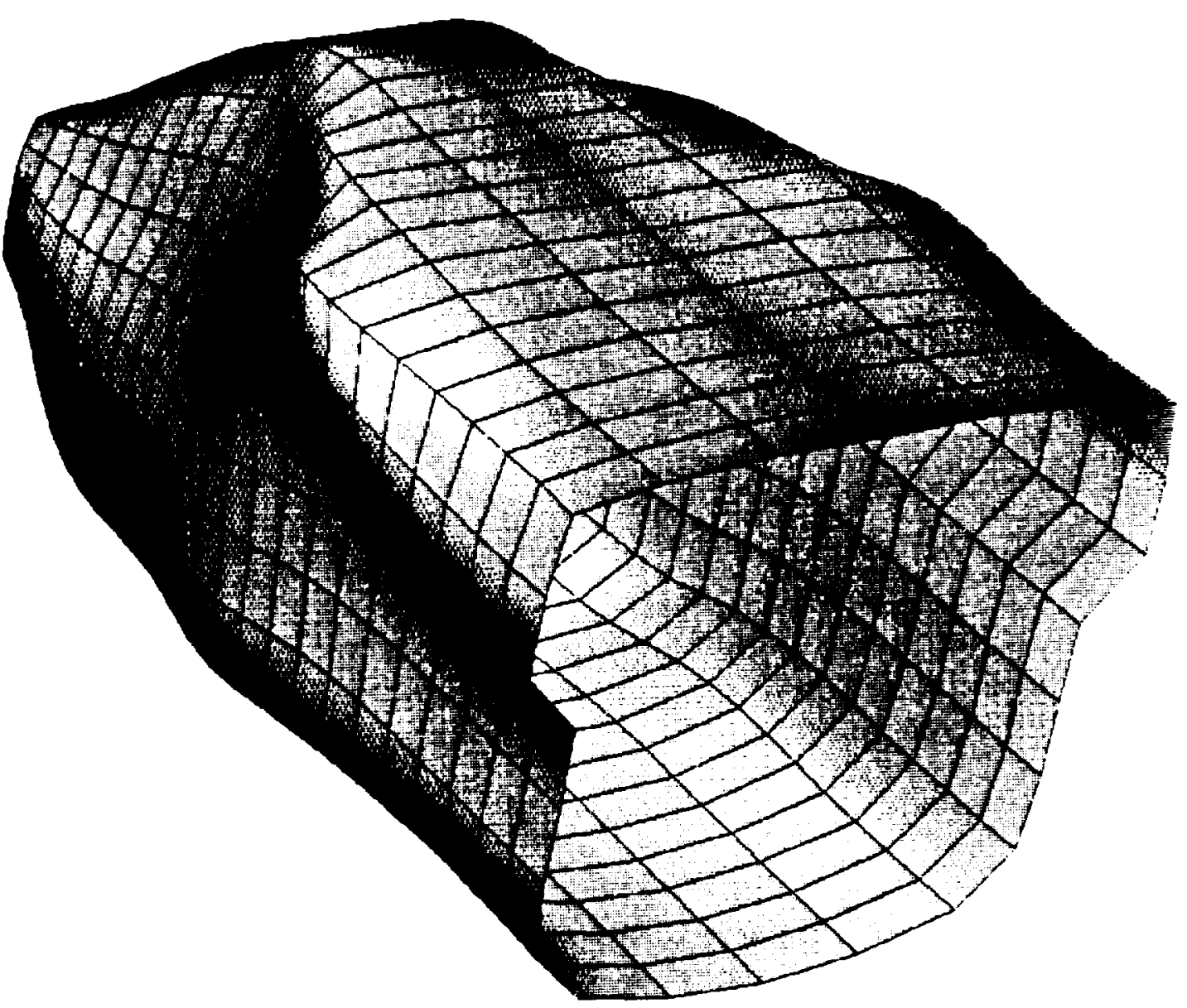

Figure 2.4: Several contour are combined to produce a continuous surface model of a portion of the LHX fuselage. 


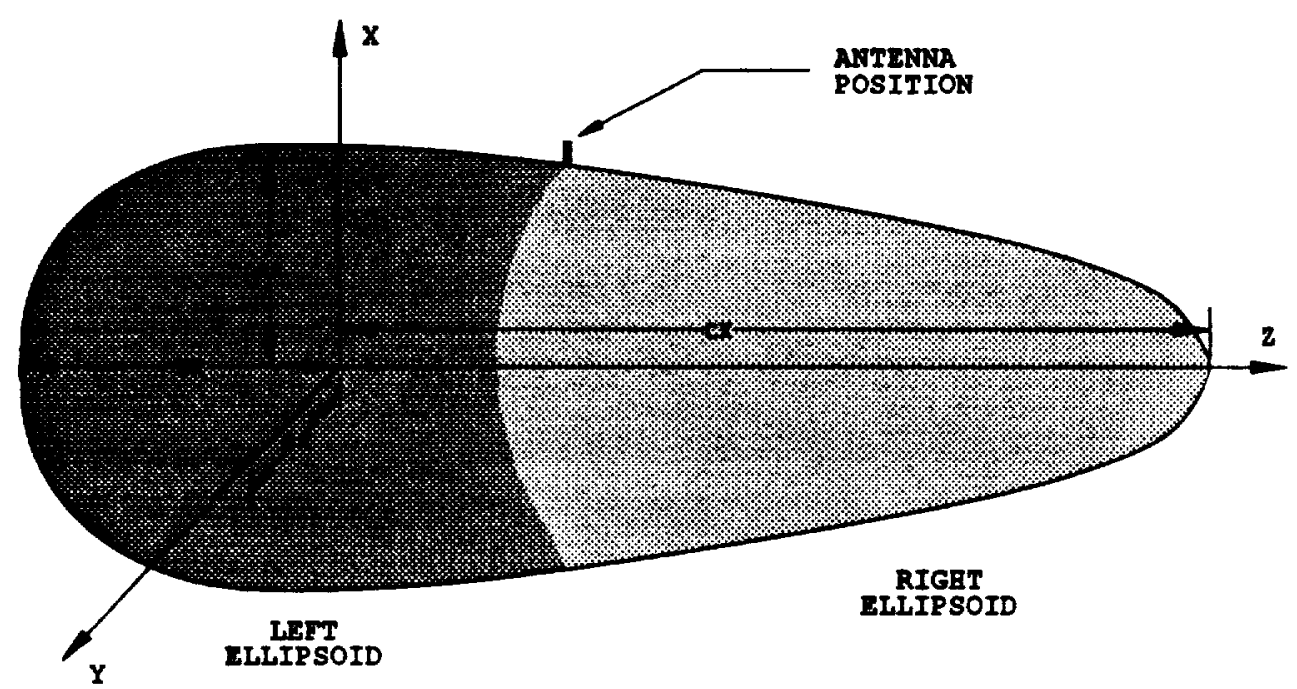

Figure 2.5: In the UTD model, the fuselage of the helicopter is modeled by two ellipsoids, joined at the antenna location. 


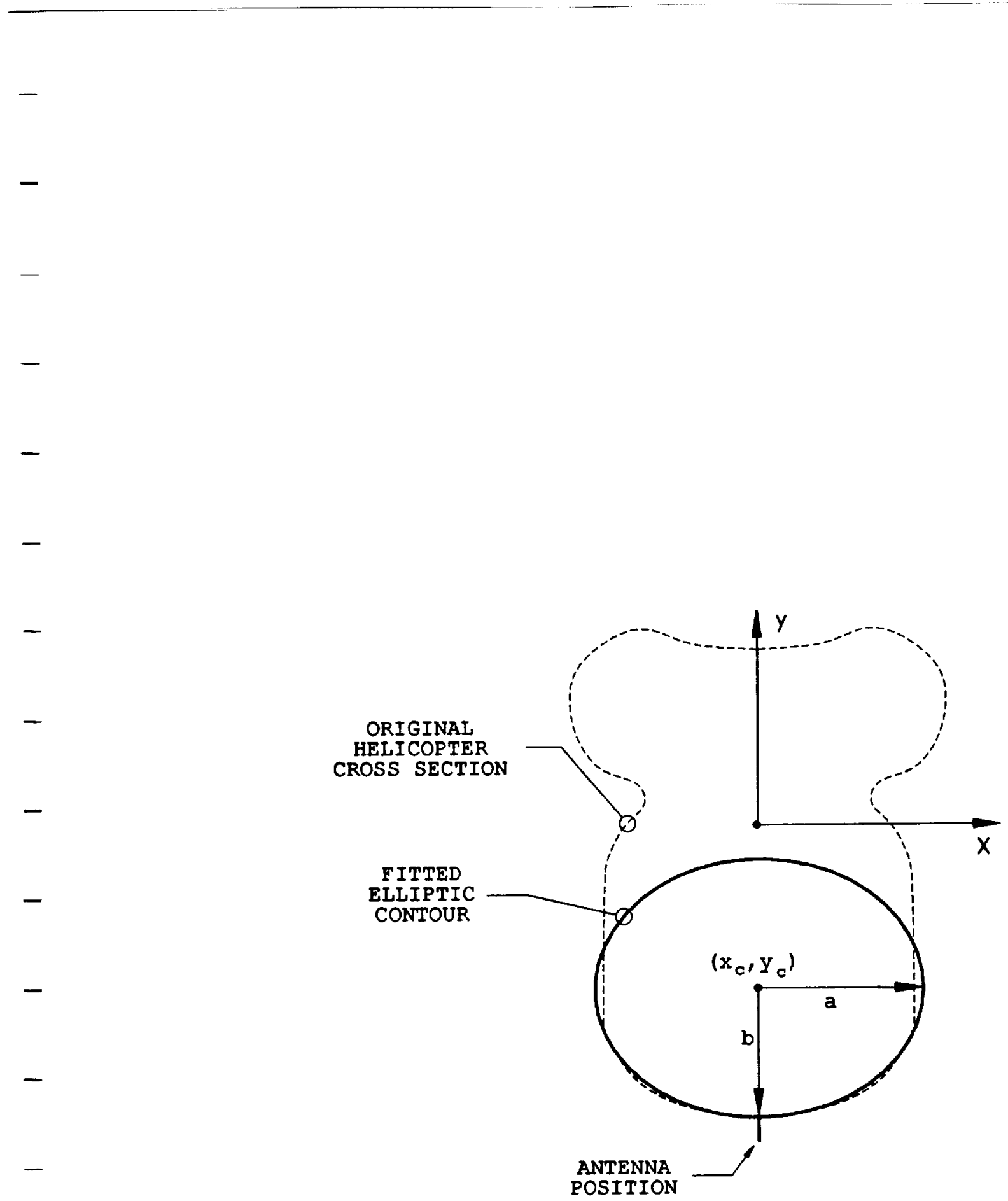

Figure 2.6: A ellipse is fit to a fuselage cross section. 


\section{Chapter 3}

\section{Aircraft Fuselage Representation}

\subsection{Introduction}

This chapter will describe a number of computer subroutines which have been developed to model the fuselage of the desired aircraft with the wings omitted. The data which defines the surface must be taken from crosssections available on the blueprint of the aircraft. The surface must be smooth, meaning that it has a continuously turning normal. Of the many possible methods of representing the surface, a tensor-product form was selected.

\subsection{Input Data from Blueprints}

A subroutine has written to input points from the blueprint of the aircraft using a digitizing puck. A point on the y-axis of the aircraft where a cross-section is available is called a y-station. Note that the y-axis is the longitudinal axis of the aircraft. 
For each $y$-station points $(x, z)$ are accepted from one half of the crosssection using the digitizing puck. The body is assumed symmetrical about the centerline. The result is displayed on the computer screen. If the crosssection does not look good the points used to represent the section may be modified.

A file is created for each $y$-station. This file includes the $y$-coordinate of the station and the $(x, z)$ coordinates describing the cross-section. A master file is created for the entire aircraft body. This file identifies the cross-sections describing the aircraft's fuselage.

\subsection{Cross-section representation}

On each cross-section a smooth curve is fit thru the $(x, z)$ points describing the section. Here $x=x_{i}(t), z=z_{i}(t)$, and $y=y_{i}=$ constant for each cross-section; the letter $i$ is the index of the $y$-stations of the cross-sections and $t$ is a parameter described below. $x(t)$ and $z(t)$ are cyclic cubic splines. The curve is smooth: the second derivatives of $x(t)$ and $z(t)$ with respect to $t$ are continuous. Points at any position on the cross-section can now be calculated. Reference [5] describes the ideas used. (Note: Taut splines were considered here but not used.)

The parameter $t$ was chosen to make the cross-sections smooth but with no ripple and to prevent spurious loops or oscillations. The parameter $t$ was generated using equation (3.1) below. Note: $s$ is arc length along the polygon the vertices of which were input from the blueprint.

$$
\Delta t_{k}=\Delta s_{k}\left(1+\frac{2 \theta^{2}}{1+r}\right)
$$


Here $\theta$ represents change in inclination of the tangent to the section, and $r$ represents change in distance between $(x, z)$ points on the section. We call this parameterization "pseudo-arc-length". Figure 3.1 shows a crosssection generated using equation (3.1) . Figure 3.1 also shows a crosssection generated using a more classic method: $\Delta t_{k}=\Delta s_{k}$. This is called "chord-length" parameterization. The parameter $t$ is normalized from 0 to 1 on each cross-section.

\subsection{Blending along $y$-axis}

Blending of the $(x, z)$-section in the $y$-direction is performed using the tensor products listed below. See Reference [9]. This is sometimes called "lofting".

$$
\begin{aligned}
& x_{3 d}=x_{3 d}(t, y)=\sum_{i=1}^{n} x_{i}(t) l_{i}(y) \\
& z_{3 d}=z_{3 d}(t, y)=\sum_{i=1}^{n} z_{i}(t) l_{i}(y)
\end{aligned}
$$

In equation (3.2) and (3.3) $\mathrm{n}=$ number of $\mathrm{y}$-stations and $l_{i}(y)$ is a natural cubic spline with $l_{i}\left(y_{j}\right)=\delta_{i j}$; here $y_{j}$ is the $\mathrm{j}$-th $\mathrm{y}$-station coordinate.

Note: for $l_{i}(y)$ several sets of basis functions were tried; these sets include linear splines, half the Hermite basis, and exponential polynomials. 


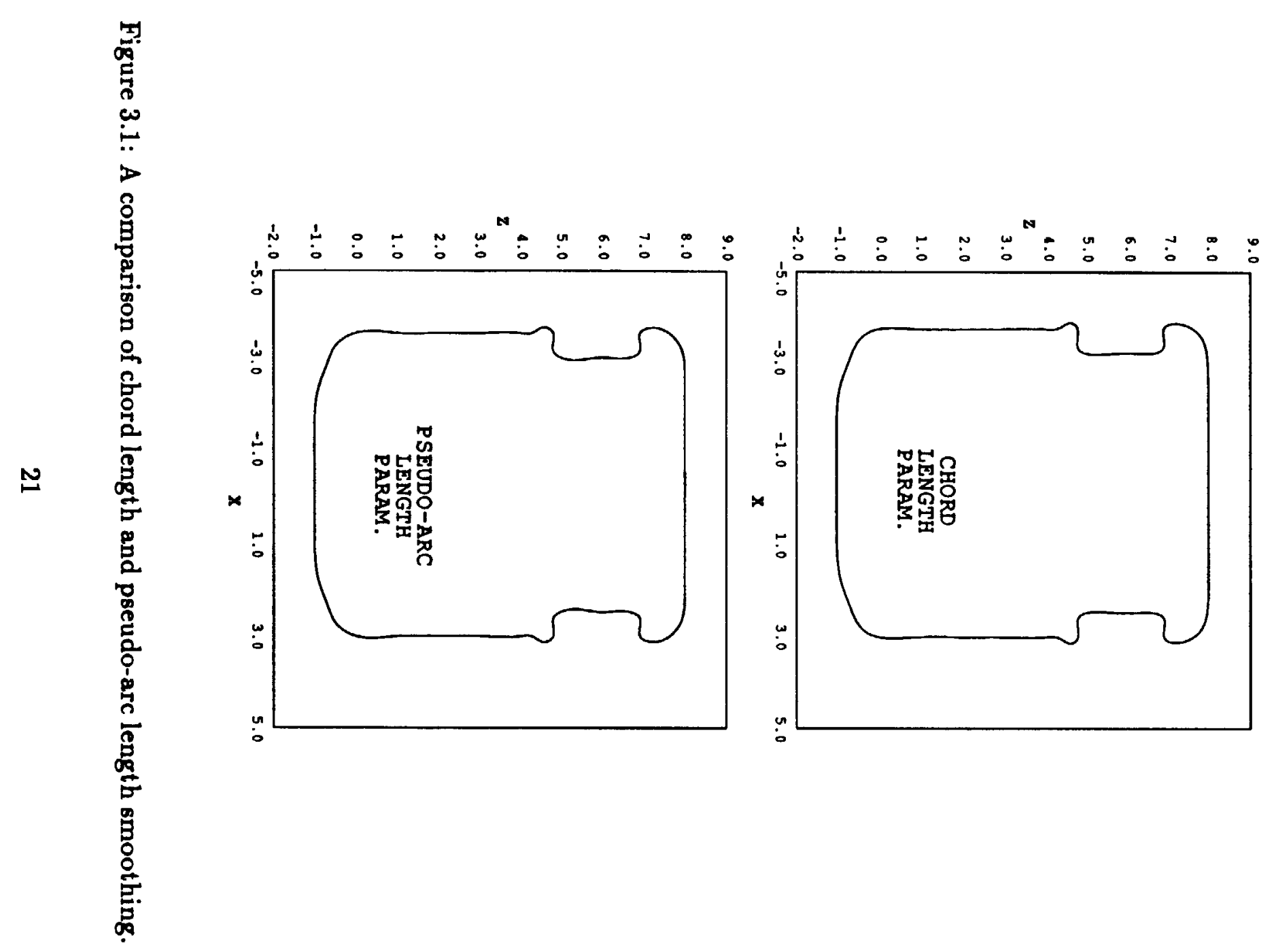




\section{Chapter 4}

\section{Moment Method Model Development}

\subsection{Moment Method Plate Model}

One of the main goals of this project is the automation of the entry of geometric data for the electromagnetic analysis of helicopters. What is implied by this, as far as the moment method is concerned, is the automated creation, from input data entered by the user, of a model in the input format required by the method of moments Aircraft Modeling Code (AMC) [22].

The AMC code is based upon the Electromagnetic Surface Patch Code: Version IV (ESP) which models general bodies by an interconnection of thin wires and polygonal plates [23]. In the case of modeling helicopters, the body of the helicopter and all its wings and fins are modeled as a collection of polygonal plates and the antenna, mounted on the helicopter, is modeled as a wire. A requirement of this software is that the polygonal plates may only contact one another at their edges.

To more clearly understand the problem at hand, it is necessary to 
understand some of the input requirements of the AMC code. The AMC program enters the aircraft geometry in the form of a set of cross sections and attached wings. Each cross section is composed of a set of points specifying a vertex on the body. Each of these vertices is then tied together to form a closed polygonal plate model or mesh of the fuselage of the aircraft. The problem occurs when fins must be attached to the body (fins here imply any type of aircraft control surface which wings, a tail, elevators, or any appendages sticking out from the body). Fins may only attach along the intersection of the polygonal plates which make up the fuselage. This places a very tight constraint on the way the points that make up the cross section of the body are chosen, and was the major problem in automating the model creation process. Essentially the problem is that the wings and fins must exactly fit into the edges of the fuselage plate grid. The problem is especially complicated when there are several fins attached to the fuselage.

A solution to this problem, for the types of geometries encountered when modeling helicopters, was formulated and successfully implemented. The resulting algorithm is, to some extent, based on the scanline algorithms used in computer graphics. Experimentation with several geometries showed that the algorithm does produce plate geometries which are compatible with the AMC program. As an illustration, Figure 4.1 shows a continuous representation of a helicopter shape with wings and fins. Figure 4.2 shows the MM plate model of this helicopter. Note that the continuous surface of the helicopter is modeled by a number of quadrilateral plates. Also note that this wings and fins exactly fit into the fuselage plate grid, as is required by AMC. The arrows in Figure 4.2 indicate the surface patch dipole modes 
used by AMC to expand the current on the helicopter.

Future directions for year two of this project call for increased automation on the part of the decomposition algorithm. The current version of the program is lacking in what might be called intelligence. What is meant by intelligence is the making of judgements by the software concerning how best to create the model, or for that matter, whether it should bother creating the model at all if factors indicate that the desired problem is too big for the moment method to practically handle. Current plans call for the addition of a simple artificial intelligence (AI) system to help in making model creation decisions.

\subsection{D Graphics}

A major appeal of modern workstation CAD packages is the ability to draw high quality shaded color images of the objects to be studied. This appeal is particularly acute when dealing with 3-D geometries such as computer models of helicopters. A high priority in the writing of this software package is the ability to display shaded 3-D models of the various helicopter geometries being manipulated and analyzed. With the advent of high speed graphics hardware facilities in modern workstations, it is possible for the user to rotate and manipulate images of the 3-dimensional objects they are analyzing in real time.

The 3-D graphics work done to date, on the Tektronix 4337 workstation, falls into three separate categories: The first is the rendering of the cross sections entered by the user as a smoothly shaded body; the second 


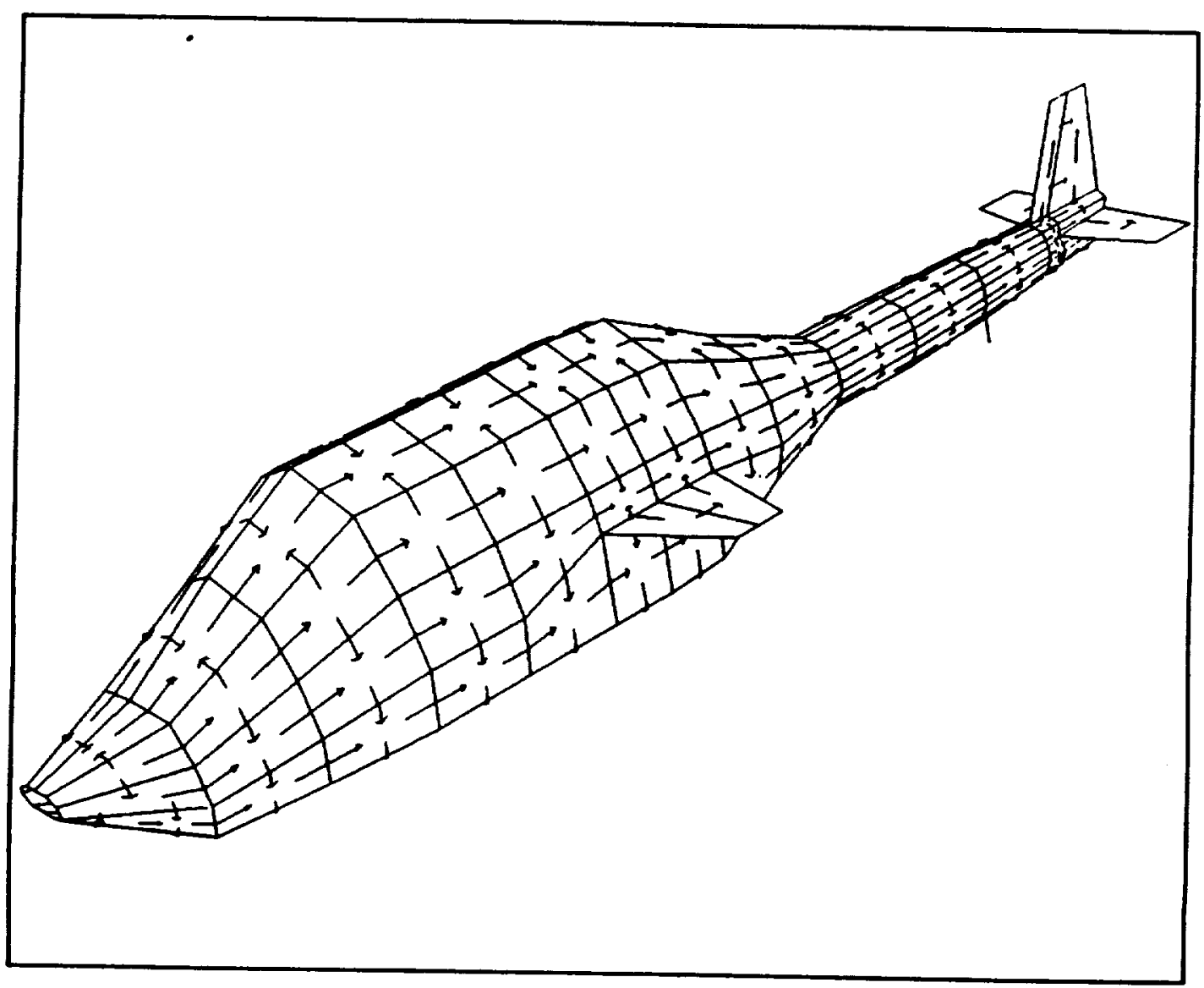

Figure 4.2: A plate model of a helicopter with arrows indicating the MM surface patch dipole modes used by AMC. 
is the rendering of the polygonal plate models used by the moment method algorithm; and, the third is the rendering of the ellipsoid and plate approximations to the body of the helicopter for the geometric theory of diffraction model.

The model of the cross sections entered by the user is performed by making a spline fit to the cross sections entered, and then rendering the splines by use of polygonal approximations and intensity interpolation (Gouraud) shading. Any fins attached to the body are modeled as flat polygonal plates. This model allows the user to visualize the results of the data they have entered and to gauge whether the shape of this model resembles the shape of the desired helicopter sufficiently to merit electromagnetic analysis.

The moment method model is created by drawing, as flat shaded polygons, all the plates which make up the fuselage and the fins that are attached to it. The resulting model is a shaded rendering of a polygonal helicopter. The purpose of this model is to allow the user to see if the plate model created is sufficiently similar to the actual helicopter that the analysis performed would be accurate.

The geometric theory of diffraction model involves the drawing of the ellipsoids and plates which make up the approximation to the surface of the helicopter. This model allows the user to see how well the geometric theory of diffraction model fits the shape of the body at the desired antenna location.

A problem with 3-D graphics is that they are machine dependent and thus difficult to transport to other systems. A program which runs on one graphics system is not very likely to run well on another. The only way to 
produce high speed (real time) graphics, is by writing programs which are very efficient on the hardware you are working with. There is also the problem of the capabilities of some graphics systems being far superior to those of others. This causes problems by not allowing a program which might do some very fancy graphics operation to be adapted onto a simpler system which cannot handle that type of operation. To alleviate this problem, all the 3-D graphics programs which are part of the HARP package interface to what we call a device independent $9-D$ graphics library. This consists of a small set of routines (eight routines) which, with little trouble, can be adapted to another 3-D graphics system. The operations chosen are common to most graphics systems, and give the individual adapting the package a great deal of freedom in the implementation of the HARP graphics programs require. The 3-D graphics routines of the HARP package call on this standard module to perform their graphics operations. When moving to a different 3-D graphics system, only this module must be modified for the particular system, none of the 3-D graphics routines of the HARP package need be changed.

The plans for graphics work for the second year of the project call for improvements to the existing 3-D software already written. The big change will occur in the integration of the 3-D graphics work already done into an $\mathbf{X}$ Windows interactive system interface. The $\mathbf{X}$ Windows interface will handle the user interaction and control of the various components of the HARP package. 


\subsection{Speeding Up the MM Analysis}

The HARP code will allow a user to interactively position an antenna at a location on a helicopter, and then to generate a radiation pattern from this antenna. It is very desirable that this process be interactive, which means that the generation of a pattern from an antenna mounted on a particular helicopter should be as fast as possible.

One method for solving this problem is the matrix MM/Green's function method for the MM solution of a two body problem [11]. In our case, the two bodies are the antenna and the helicopter. As originally presented by Newman, the matrix MM/Green's function technique required the inversion of the body 2 (helicopter) block of the MM impedance matrix. For large matrices, matrix inversion is a very time consuming numerical procedure, and thus the matrix MM/Green's function method was slow. However, we have re-formulated the matrix MM/Green's function solution so that it requires only an $\mathrm{LU}$ decomposition of the body 2 block of the impedance matrix. Since an LU decomposition is much faster than a matrix inversion, the matrix MM/Green's function procedure is much faster. For example, in one particular run we reduced the computation time from approximately thirty minutes to two minutes. 


\section{Chapter 5}

\section{GTD Model Development}

In the high frequency regime, the analysis of the radiation of an antenna(s) mounted on the fuselage of a helicopter will be carried out by means of a computer code referred to as the AIRCRAFT CODE. This code, was developed at the Ohio State University ElectroScience Laboratory, and is based on the uniform version of the Geometrical Theory of Diffraction, also known as the GTD or UTD technique. This implies that in order to use this code, it is necessary to generate input data (from whatever information is available about the helicopter) which is compatible with the input data of the Aircraft Code. Recall that the Aircraft Code models the helicopter by a composite ellipsoid and a set of flat plates. Thus, the initial effort of the GTD group's research deals with attempting to fit a composite ellipsoid to the fuselage of a helicopter. A composite ellipsoid is formed by joining two ellipsoids at the antenna location as shown in Figure 5.1. Once this is done, the next stage will be to work on plate at tachments to the ellipsoid obtained so as to enable the utilization of these geometrical results as input data to the now available Aircraft Code. 


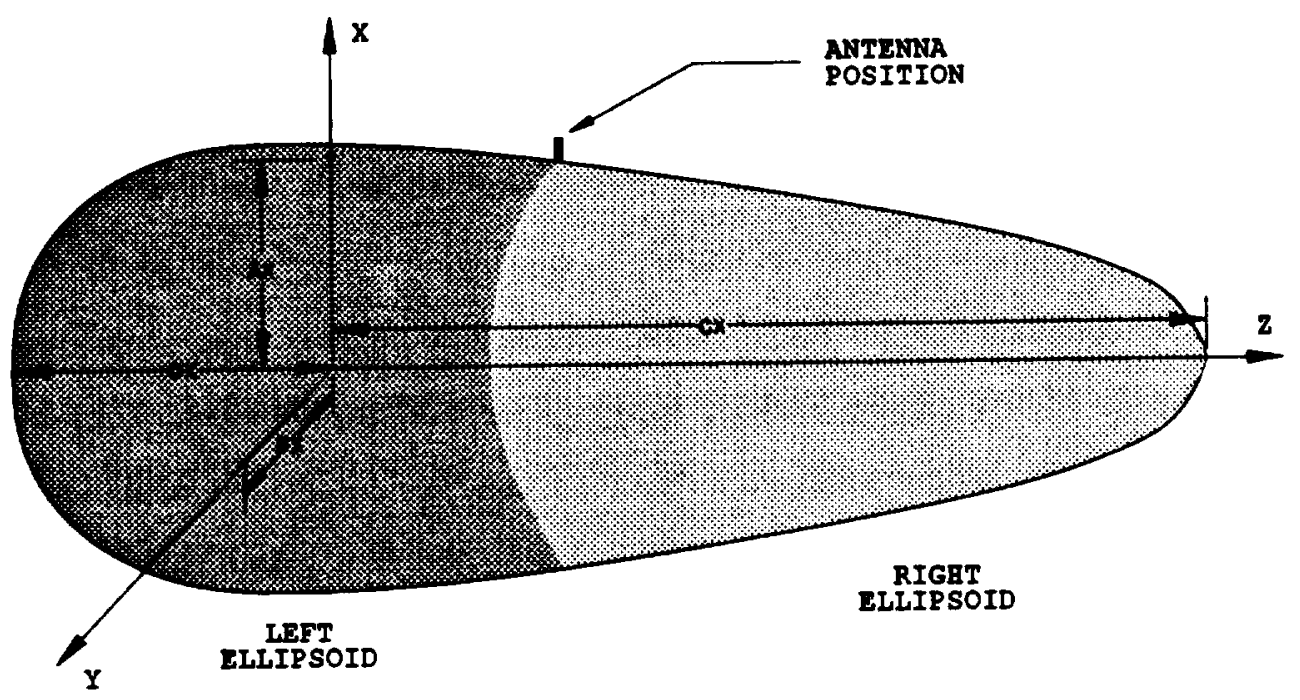

Figure 5.1: A composite ellipsoid.

There are a number of ways that an ellipsoid can be fitted to a certain region of the fuselage, but regardless of what procedure is followed, the final result is a set of four parameters which completely describe the composite ellipsoid as shown in Figure 5.1. It is very important to keep in mind that, in general, the composite ellipsoid will fit only a localized region of the helicopter fuselage in the vicinity of the antenna location. In other words, when we talk about fitting a composite ellipsoid to the fuselage of the helicopter, we are talking about local fitting in the vicinity of the radiating antenna, in contrast to global fitting.

The four parameters of the composite ellipsoid can be determined by either solving the three-dimensional problem directly or solving two twodimensional problems. The latter method was followed in our research. 
That is, first the cross section of the fuselage where the antenna is located and which is perpendicular to the axis of the fuselage is considered. In this cross-section, an ellipse is fit to the true cross-section of the helicopter. This particular ellipse can have its center shifted from the origin of the coordinate system, but it is not rotated. This means that four parameters have to be determined as depicted in Figure 5.2, namely, the coordinates of the center of the ellipse and its semi major and minor axes. The next step is to consider a second cross-section of the fuselage which passes along the antenna location and it is perpendicular to the first one. This two-step procedure will allow us to determine the four parameters of the composite ellipsoid.

To date, the fitting of an ellipse to the fuselage cross-section perpendicular to its axis has been considered. Once this step is completed, the next step, namely the fitting of the the fuselage cross section perpendicular to the first one, will be easily done. Although the problem of fitting an ellipse to a two-dimensional cross-section appears to be a simple problem, we found in the course of our research that there are a number of difficulties with the convergence of the solution which is obtained numerically. In general, it appears that the solutions are very dependent in how the problem is posed. In other words, it appears that we are dealing with an ill-posed problem. Several approaches were followed where each approach has some advantages and disadvantages over the others. This report discusses the different approaches that have been considered and the problems that were encountered. 


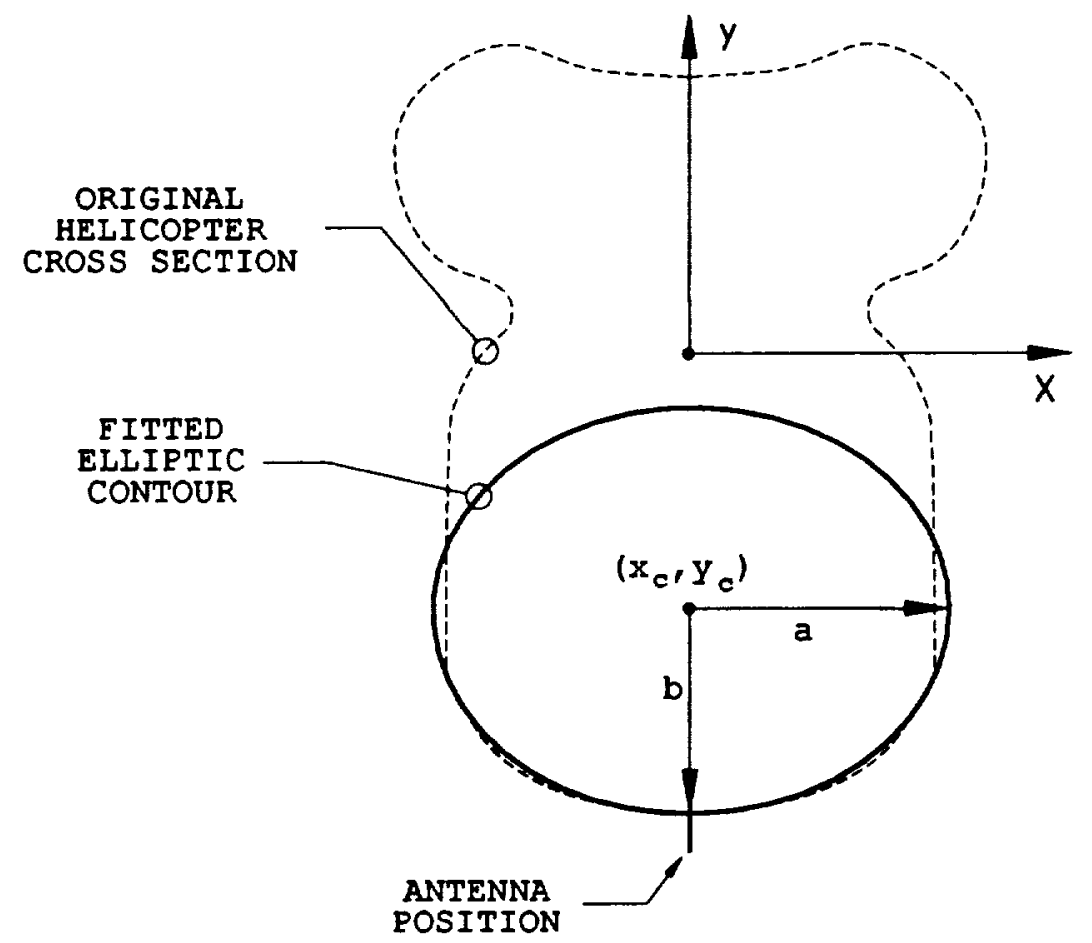

Figure 5.2: The parameters which define a shifted ellipse. 


\subsection{Method 1 - Four Nonlinear Equations}

In this approach, the fours parameters of the ellipse (see Figure 5.2) are obtained by solving four simultaneous nonlinear equations. There are many equations from which a set of four independent equations can be obtained. However, before the equations can be obtained, it is necessary to determine what is known. The known parameters are: (1) the antenna location and (2) the coordinate points of the entire fuselage cross section. Based on this information, one can obtain additional information about the cross-section such as the outer normal, tangent vector, and curvature of this cross section at the antenna location.

The ellipse shown in Figure 5.2 can be described by the following equation

$$
\left(\frac{x-x_{c}}{a}\right)^{2}+\left(\frac{y-y_{c}}{b}\right)^{2}=1
$$

where $\left(x_{c}, y_{c}\right)$ are the coordinates of the center of the ellipse and $a$ and $b$ are its semi major and minor axes, respectively. An alternative equation which is more convenient to calculate the normal, tangent and curvature vectors is as follows:

$$
\vec{R}\left(V_{r}\right)=\hat{x}\left(a \cos V_{r}+x_{c}\right)+\hat{y}\left(b \sin V_{r}+y_{c}\right)
$$

where

$$
\tan \phi=\frac{b \sin V_{r}+y_{c}}{a \cos V_{r}+x_{c}}
$$

From Equations (2a) and (2b), the tangent $(\hat{t})$ and curvature $(\hat{k})$ vectors 
at the antenna location $\left(x_{a}, y_{a}\right)$ can be easily obtained, namely

$$
\begin{array}{cc}
\hat{t}=\hat{x} t_{x}+\hat{y} t_{y} ; \quad t_{x}=\frac{-a^{2}\left(y_{a}-y_{c}\right)}{M} ; \quad t_{y}=\frac{b^{2}\left(x_{a}-x_{c}\right)}{M} \\
\vec{k}=\hat{x} k_{x}+\hat{y} k_{y} ; \quad k_{x}=\frac{-a^{4} b^{6}\left(x_{a}-x_{c}\right)}{M^{4}} ; \quad k_{y}=\frac{-a^{6} b^{4}\left(y_{a}-y_{c}\right)}{M^{4}}
\end{array}
$$

and

$$
|\vec{k}|=K=\frac{(a b)^{4}}{M^{3}}
$$

where

$$
M=\left[a^{4}\left(y_{a}-y_{c}\right)^{2}+b^{4}\left(x_{a}-x_{c}\right)^{2}\right]^{1 / 2}
$$

From the set of equations given above, four independent nonlinear equations can be chosen. The first set of equations that were used were Equation (1) evaluated at the antenna location $\left(x_{a}, y_{a}\right)$ and at $\left(x_{2}, y_{2}\right)$, a point in the neighborhood of $\left(x_{a}, y_{a}\right)$, Equation (3c) and the equation for $t_{x}$, the $x$-component of the unit tangent vector in (3a). It is important to keep in mind that the two components of the unit tangent vector are not independent of each other, so only one of the two components can be used. Once the four equations are chosen, we can now proceed to solve this system of four nonlinear equations for the four unknowns: $x_{c}, y_{c}, a$ and $b$. A commonly used technique is the Newton-Raphson Method which requires a good initial guess of the solution. That is, if the initial guess is close to the true solutions, this method converges very fast. Otherwise, it will usually not converge. The main difficulty with this method is then to obtain a good 
initial guess which sometimes has to be obtained by other techniques, such as the Steepest Descent technique. A code utilizing this method [12] was developed and tested. Note that two different $L U$ decomposition subroutines for solving the matrix equation encountered in the Newton-Raphson code were attempted: (1) the subroutine developed by ESL, and (2) using a software package known as LINPACK [13]. Both routines, however, gave similar results. It was found that for the cases we considered, the NewtonRaphson code does not converge at all when the initial guess values for the roots (the solution) were not within approximately $4 \%$ of the exact solution.

Hence, a more global approach is necessary which will converge to the neighborhood of the exact solution even if the initial guess is not very good. One such technique is the Steepest Descent method. This method determines a local minimum in which a multi-dimensional system (in the present case of dimension four) is transformed into a single dimensional form [14]. Nevertheless, this technique is relatively slow compared to the Newton-Raphson Method, but is in general global, i.e. if there is a solution, it will converge to the vicinity of the exact solution as long as enough iterations are performed. However, as the number of iterations increases, the round off errors also increase. Thus, it was decided that the Steepest Descent Technique would be used to converge the initial guessed solutions to an accuracy whereby it would certainly be accepted as input initial guessed solutions for the Newton-Raphson Method. This would expedite the obtaining of the solution in general.

For the system of equations consisting of two ellipse equations, the cur- 
vature equation, and the $x$-component of the tangent vector equation, the Steepest Descent Technique solution converges to less than $6 \%$ of the exact solution. Since the Newton-Raphson Method in general accepts initial guessed values of approximately not more than $4 \%$ error, the solution generated by the Steepest Descent Technique was unusable for this purpose. So, it was decided exclusively that only the Steepest Descent Technique could be employed. It must be noted that, however, this technique was not fail proof. In general, of course, the code converged to $6 \%$ of exact solution for iterations of not more than 1500 times. Occasionally, however, in some test cases run with this technique, it failed to converge to the proper solution if the initial guessed values were off by approximately more than $30 \%$.

The speed of convergence strongly depends in the type of function being minimized. This multivariable function, commonly referred to as the cost function, is given by

$$
G\left(f_{1}, f_{2}, f_{3}, f_{4}\right)=\sum_{i=1}^{4} f_{1}^{2}
$$

where

$$
\begin{gathered}
f_{i}=\left(\frac{x_{i}-x_{c}}{a}\right)^{2}+\left(\frac{y_{i}-y_{c}}{b}\right)^{2}-1 ; \quad i=1,2 \\
f_{3}=K-\frac{(a b)^{4}}{M^{3}} \\
f_{4}=t_{a}+\frac{a^{2}\left(y_{a}-y_{c}\right)}{M}
\end{gathered}
$$




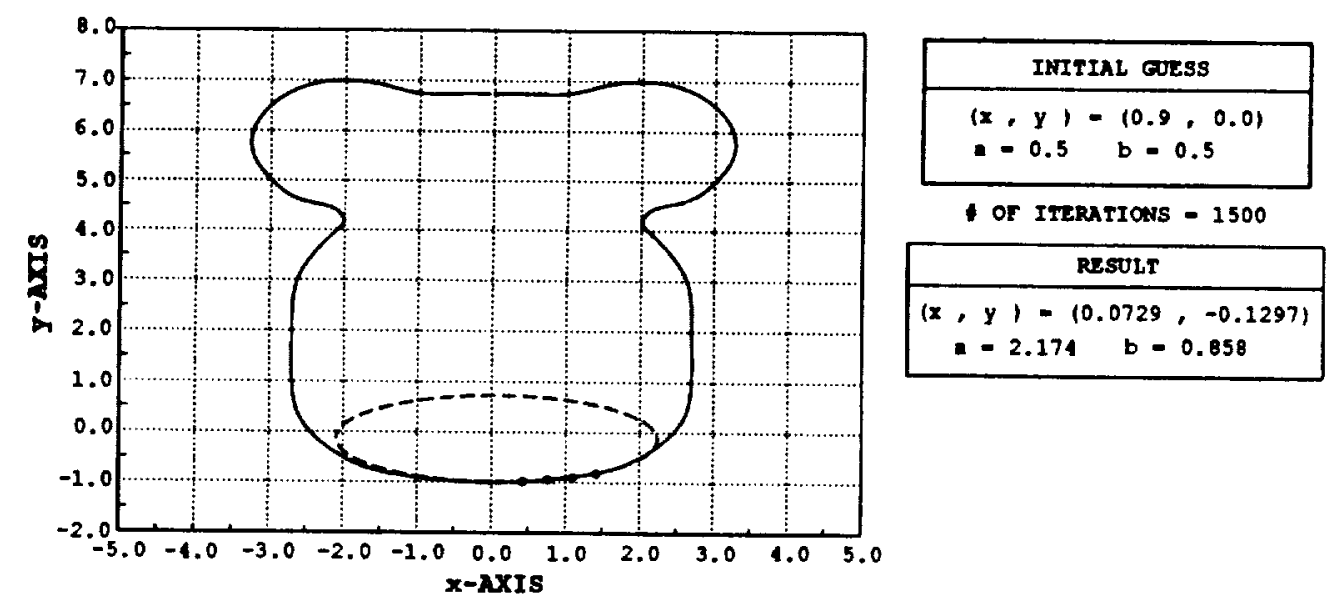

Figure 5.3: A four point fit by means of the SDA.

where $x_{1}=x_{a}$ and $y_{1}=y_{a}$. It turns out that this particular cost function is very flat in the region where its minimum is located which implies that the Steepest Descent technique would have difficulty to detect it. Thus, it is necessary to choose another set of equations such that the resulting cost function would not have the property described above. The second set of equations that was tested consisted of four ellipse equations, namely,

$$
f_{i}=\left(\frac{x_{i}-x_{c}}{a}\right)^{2}+\left(\frac{y_{i}-y_{c}}{b}\right)^{2}-1 ; i=1,4
$$

To ensure that the code was properly running, an actual sample of a helicopter cross section was used. In Figures 5.3-5.5 examples are shown where for a given set of four points on the cross-section and the initial guess of the parameters of the ellipse, the Steepest Descent algorithm (SDA) will calculate a new set of parameters after a number of iterations. It appears that with the present cost function, this algorithm will output the 

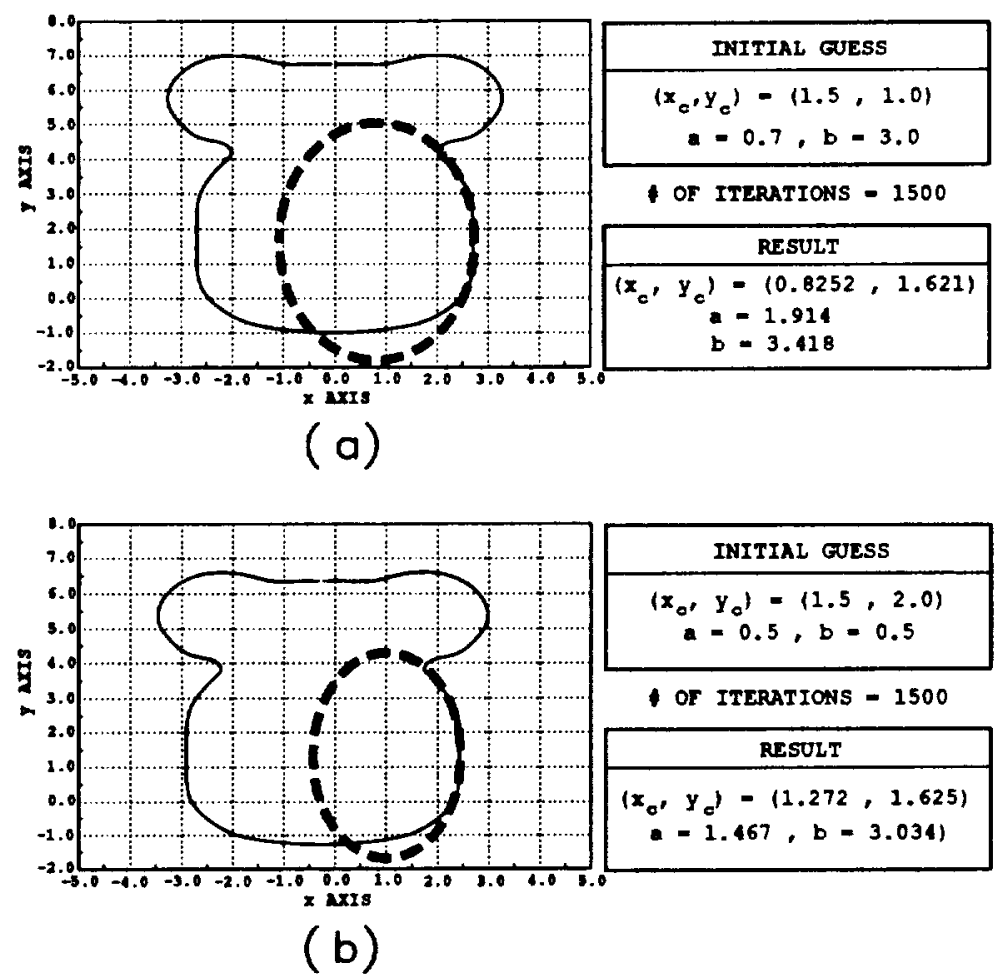

Figure 5.4: A four point fit by means of the SDA. 

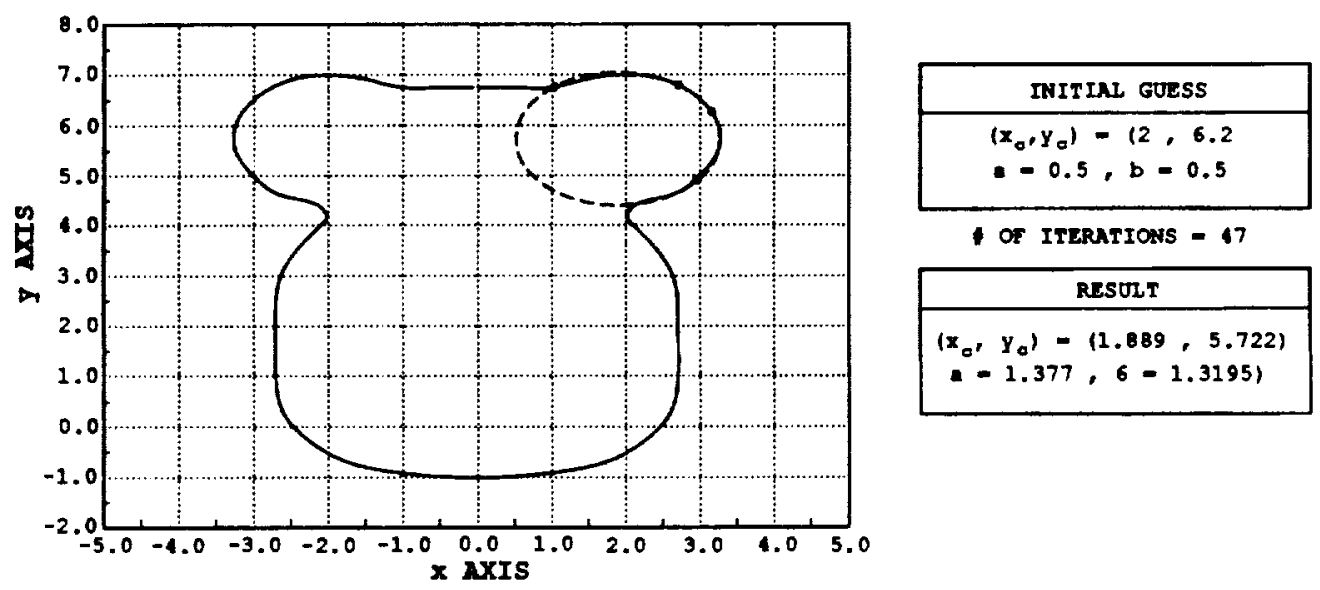

- OF ITERATIONS - 17

\begin{tabular}{|c|}
\hline REsUT \\
\hline$\left(X_{0}, Y_{0}\right)=(1.889,5.722)$ \\
$\left.y^{-} 1.377,6=1.3195\right)$
\end{tabular}

Figure 5.5: A four point fit by means of the SDA.

parameters $a$ and $b$ in such a way that the ellipse passes through or very near the four points on the cross section, regardless of the initial guess. However, it will calculate a new center of the ellipse $\left(x_{c}, y_{c}\right)$ which is not very far from the initial guess. In other words, the result depends strongly on the initial guess of $\left(x_{c}, y_{c}\right)$ but, not in the inital guess of $a$ and $b$. This can be seen more clearly in Figures 5.6-5.8. For the three cases shown in Figures 5.6-5.8, the same set of four points on the cross section are used; however, for each case, the initial guess of the four ellipsoid parameters is different. This result illustrates the fact that regardless of the initial guess of $a$ and $b$, the algorithm will calculate a new pair of values such that the new ellipse approximates the cross section very well in the vicinity of the antenna location. However, the result seems to depend on the choice of $\left(x_{c}, y_{c}\right)$. For purposes of illustration, in Figure 5.8, the initial guess of $\left(x_{c}, y_{c}\right)$ is outside the cross section, which is not a resonable guess. The 


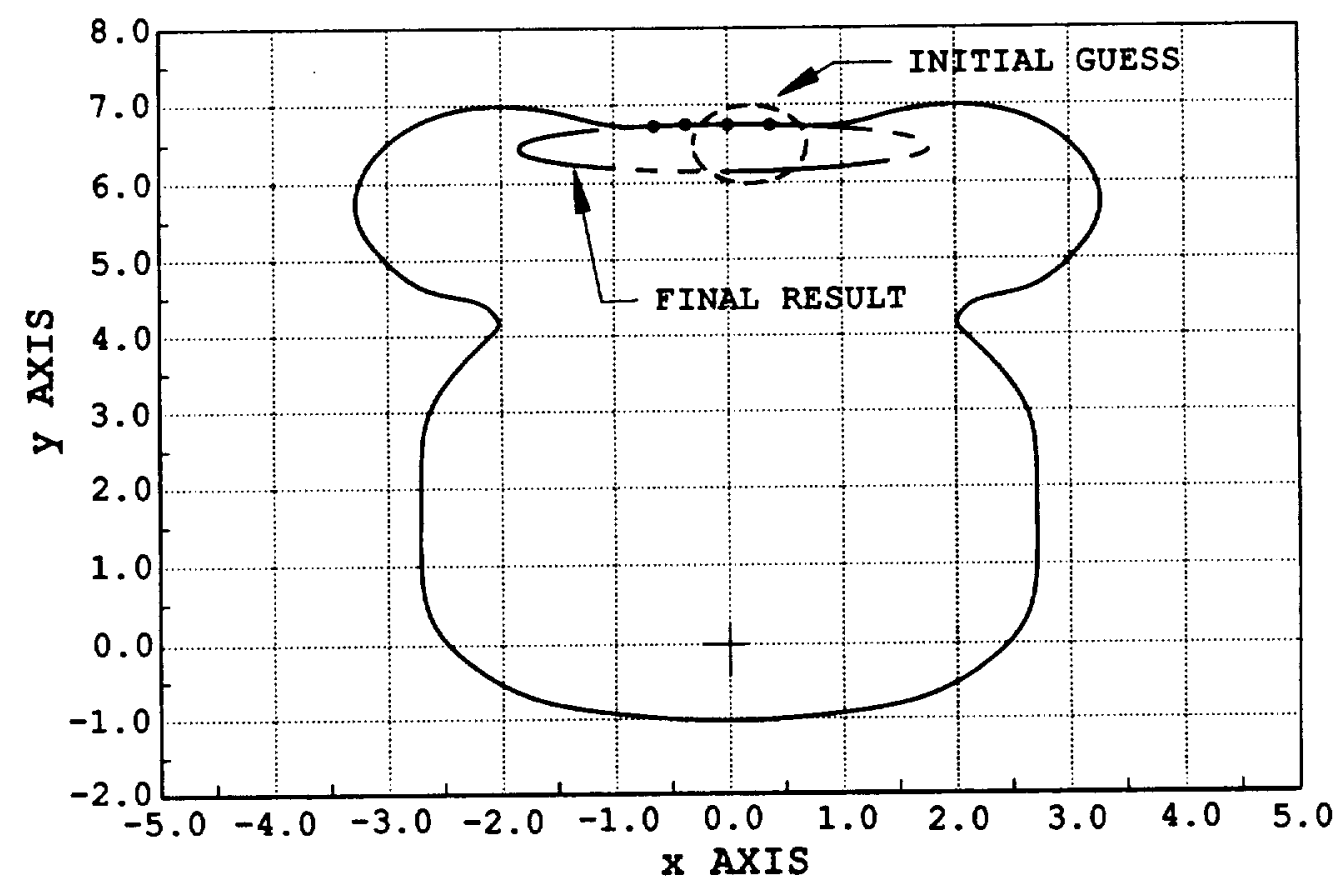

Figure 5.6: A four point fit by means of the SDA. 


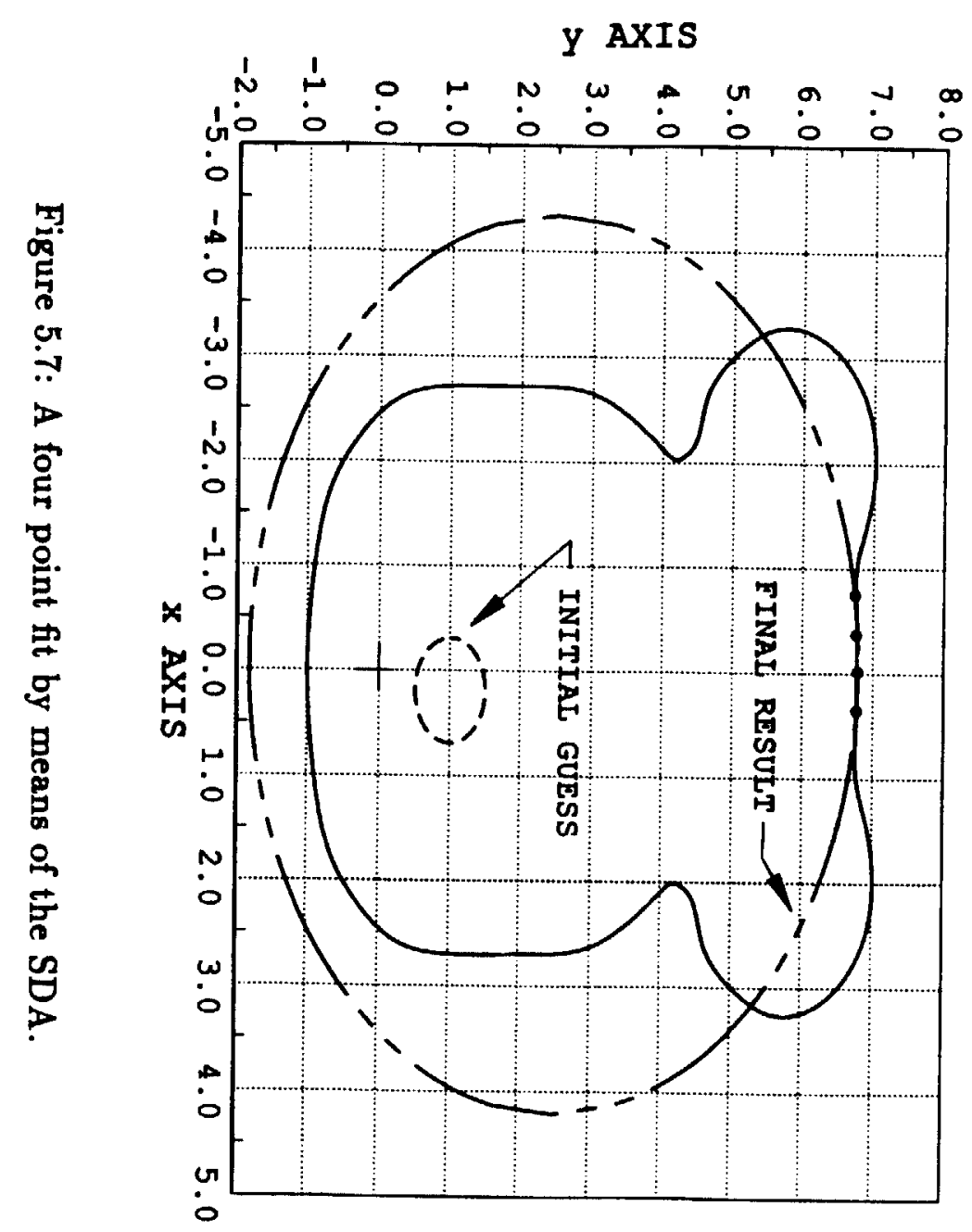




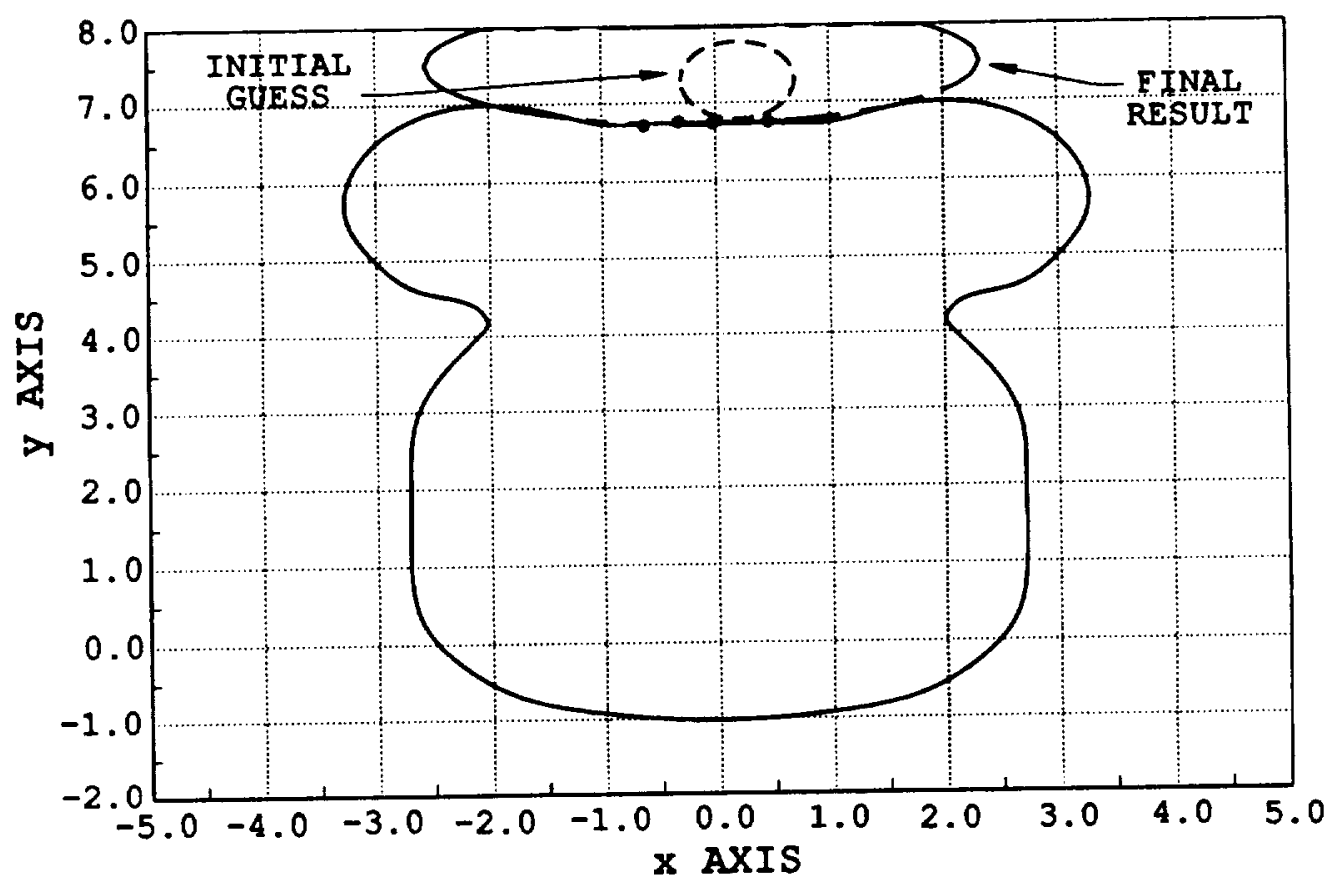

Figure 5.8: A four point fit by means of the SDA. 
calculated coordinates of the center of the new ellipse are also located outside the cross-section. Therefore, in order to ensure that the center of the ellipse is in the correct side of the cross section curve, the initial guessed values must be such that $\left(x_{c}, y_{c}\right)$ is located on the proper side of the cross section curve.

To correct the defficiency of the Steepest Descent method, namely, its slow convergence, a modified Newton-Raphson algorithm which does not require a very good initial guess was implemented. This modified version will be discussed in conjunction with Method 3. Furthermore, two other techniques which are similar to the Steepest Descent technique, namely, the Conjugate Gradient and the Powell-Fletcher algorithms [15] will also be tested in the future. In general these two later algorithms converge faster than the Steepest Descent algorithm.

For the case where the cross section curve is concave in, the calculated ellipse did not fit the region within the proximity of the antenna location at all, regardless of how the points were selected. The "best" result obtained for this case was when all the points on the cross section were chosen very close to each other. Such a case resulted in a ellipse that was tangential (or very nearly tangential) to the curve section containing at least one of those points. It is important to keep in mind that this location, although of theoretical interest, it is not expected to be a region where an antenna will be installed.

Finally, for the sake of observing some significant change in the solution, double precision was implemented in the code for the four-point system. Several cases were run with no significant change in the solutions. 


\subsection{Method 2 - N Linear Equations}

A logical extension of Method 1 discussed above is to use more than four points of the cross-section of the fuselage. Assuming that $N(N>4)$ is the number of points on the cross-section, a set of $N$ equations and 4 unknowns has to be solved. This is an overdetermined system and the well known Least Squares procedure will be used. In order to have a linear system of equations, the equation of the ellipse has to be written differently, namely

$$
A x^{2}+B y^{2}+C x+D y=1
$$

where $A, B, C$ and $D$ are the unknown coefficients. Note that the coefficients of Equation (1) can be written in terms of the coefficients of (6), namely,

$$
\begin{array}{cc}
a^{2}=\frac{E}{A} & b^{2}=\frac{E}{B} \\
x_{c}=-\frac{C}{2 A} & y_{c}=-\frac{D}{2 B}
\end{array}
$$

where

$$
E=1+\frac{C^{2}}{4 A}+\frac{D^{2}}{4 B}
$$

Equation (6) is the general equation of a quadratic surface, that is, depending on the signs of the unknown coefficients, it represents an ellipse $(A \cdot B>0)$, a hyperbola $(A \cdot B<0)$, or a parabola $(A=0, B \neq 0)$ or $(A \neq 0, B=0)$. One disadvantage of the formulation above is that this 
method does not guarantee that the result will be an ellipse because it could very well yield a parabola or a hyperbola.

The linear system of equations that needs to be solved can be written as follows:

$$
\left[\begin{array}{cccc}
x_{1}^{2} & y_{1}^{2} & x_{1} & y_{1} \\
x_{2}^{2} & y_{2}^{2} & x_{2} & y_{2} \\
\vdots & \vdots & \vdots & \vdots \\
x_{N}^{2} & y_{N}^{2} & x_{N} & y_{N}
\end{array}\right]\left[\begin{array}{c}
A \\
B \\
C \\
D
\end{array}\right]=\left[\begin{array}{c}
1 \\
1 \\
\vdots \\
1
\end{array}\right]
$$

where $\left(x_{i}, y_{i}\right)$ are the given points on the cross-section curve being fitted by a quadratic surface defined in (1) and $N$ is the number of points. To solve the overdetermined system of equations (when $N>4$ ), the LINPAK package was used. Note that with this Least Squares method (LEM) it is not necessary to have an initial guess of the parameters of the ellipse as was the case in Method 1. Two examples are shown in Figures 5.9 and $\mathbf{5 . 1 0}$ where $N=5$ and the points on the surface are indicated by the small circles. The antenna location is the center point. As mentioned several times before, the fitting is local (in the neighborhood of the antenna) and not global.

This method was tested for several antenna locations around the cross section depicted in Figure 5.11 for $N=5$ or 7 . Unfortunately, at several locations around this cross-section, the parameters $A, B, C$, and $D$ calculated by the Least Squares method yielded a hyperbola instead of an ellipse. In some other cases, no solution was found. Figure 5.11 shows points where ellipses, hyperbolas or undetermined cases were found. The labels $\mathrm{E}, \mathrm{H}$ and $\mathrm{U}$, denote, ellipse, hyperbola and undetermined case, respectively. These results depend on the number of points on the surface and 


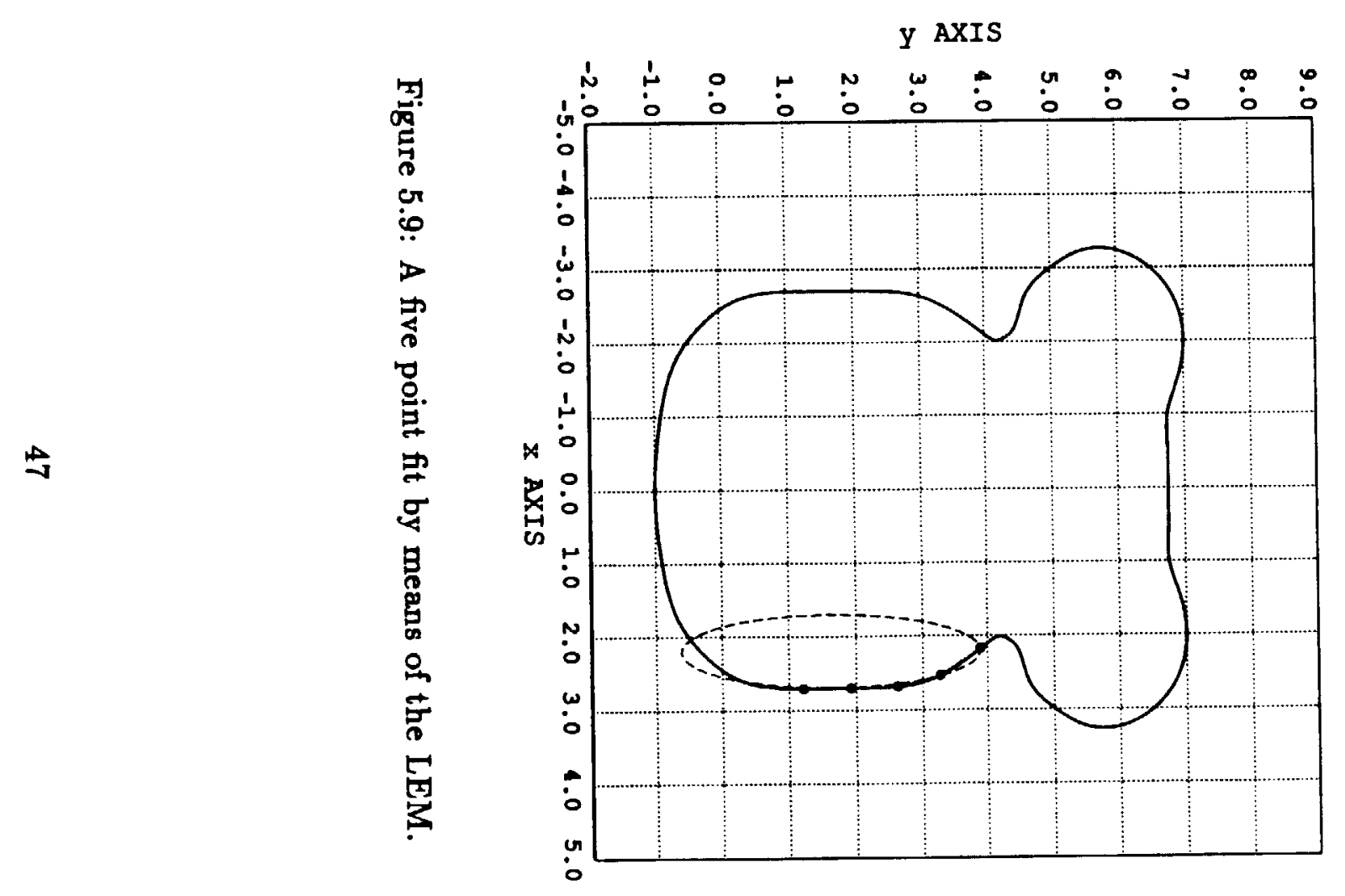




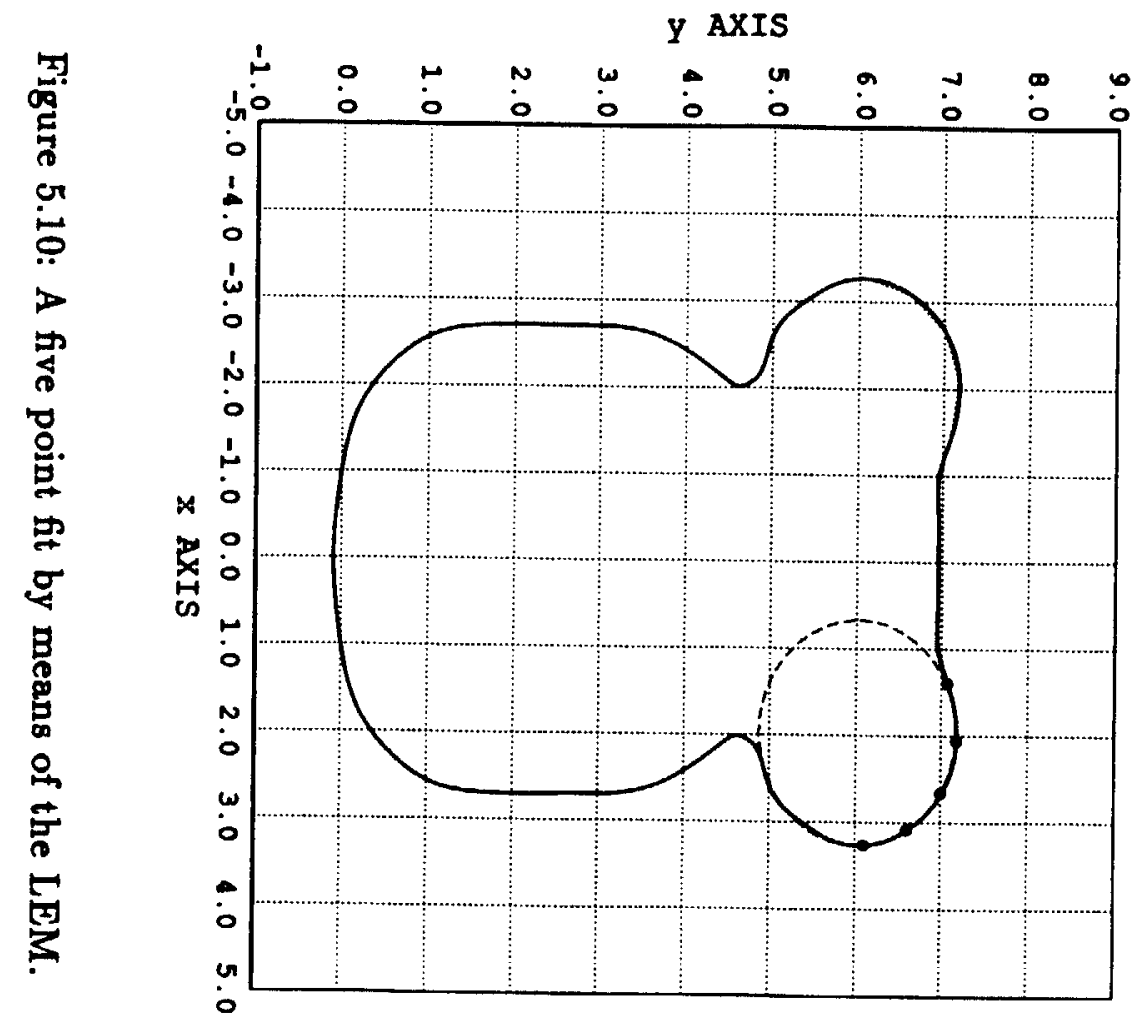




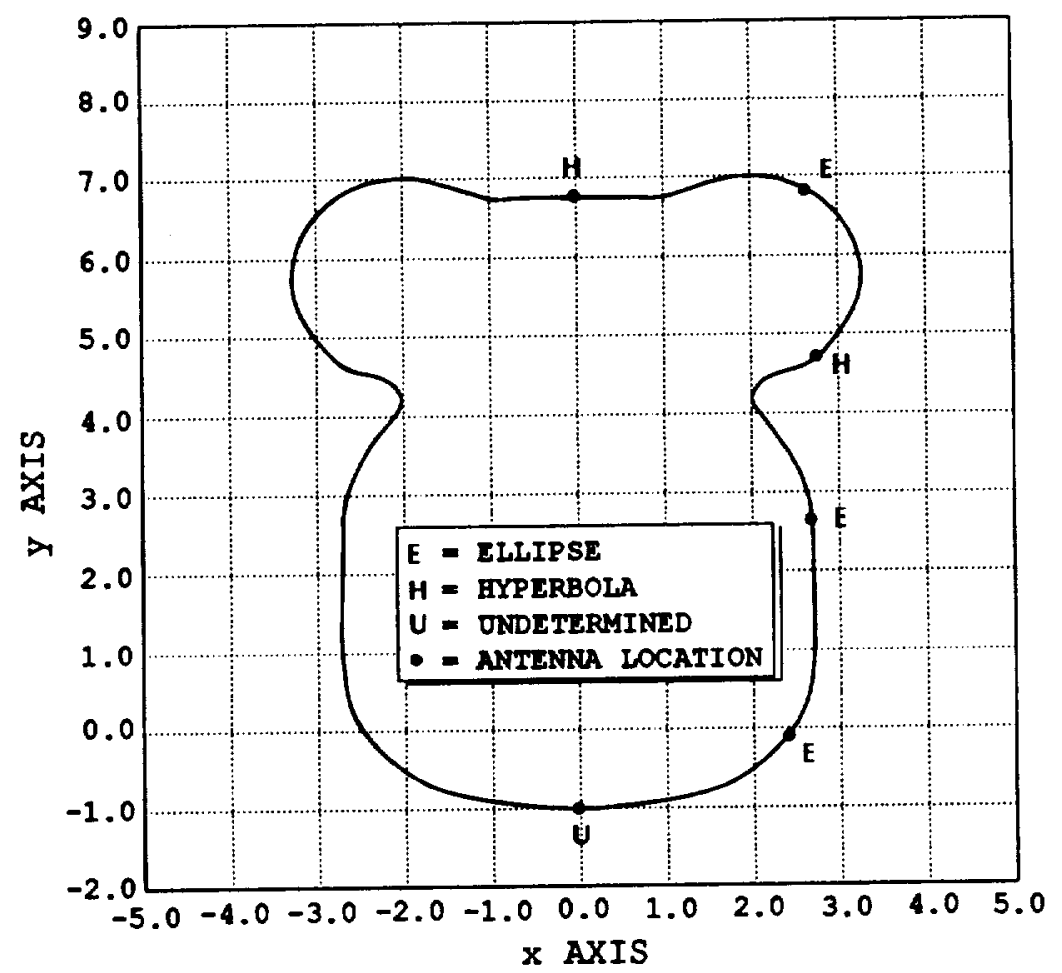

Figure 5.11: Results obtained with a five point fit for various antenna locations.

their location. Presumably, at the locations were hyperbolas were found, it may be possible to obtain ellipses by choosing the points properly.

\subsection{Method 3 - Method 1 with Penalty Func- tion}

Another technique somewhat related to Method 1 was also tried. In this case, the equation given in (1) is used to represent the ellipse. Although 
not shown here, the minimization procedure describe in Method 1 may yield values of $a^{2}$ and $b^{2}$ that are negative or zero. When this happens, the quadratic surface is no longer an ellipse. To avoid this problem, one can add a function, usually referred to as Penalty function, which will force the values of $a^{2}$ and $b^{2}$ to always be positive. There is not a unique way to define this function. However, it should be zero (or a very small positive number) when its argument is larger than a prespecified number, and a large positive number when its argument is less than a prespecified number. In this study the cost function was defined as follows:

$$
P(z)= \begin{cases}-e_{1}\left(\frac{z-e_{2}}{e_{2}}\right)^{2} & ; z<e_{2} \\ 0 & ; \text { otherwise }\end{cases}
$$

where $e_{1}$ and $e_{2}$ are constants that should be specified by the user. This Penalty function was selected to have a continuous second derivative as shown in Figure 5.12 where $e_{1}=1$ and $e_{2}=0.1$.

The cost function can then be defined as follows

$$
\psi=\frac{1}{2} \sum_{i=1}^{N}\left\{\left(\frac{x_{i}-x_{c}}{a}\right)^{2}+\left(\frac{y_{i}-y_{c}}{b}\right)^{2}-1\right\}^{2}+P\left(a^{2}\right)+P\left(b^{2}\right)+P(R N)
$$

where $N$ is the number of points and $\left(x_{c}, y_{c}\right)$ is the center of the ellipse. Note that one of the points in (10) corresponds to the antenna location $\left(x_{a}, y_{a}\right)$. The parameter $R N$ is the dot product of two vectors, namely

$$
R N=\hat{n} \cdot \vec{r} ; \quad \vec{r}=\hat{x}\left(x_{a}-x_{c}\right)+\hat{y}\left(y_{a}-y_{c}\right)
$$

where $\hat{n}$ is the outer normal to the surface at the antenna location and $\vec{r}$ is the vector from the center of the ellipse to the antenna location. As mentioned above, the Penalty function will force the values of $a^{2}, b^{2}$ and $R N$ 


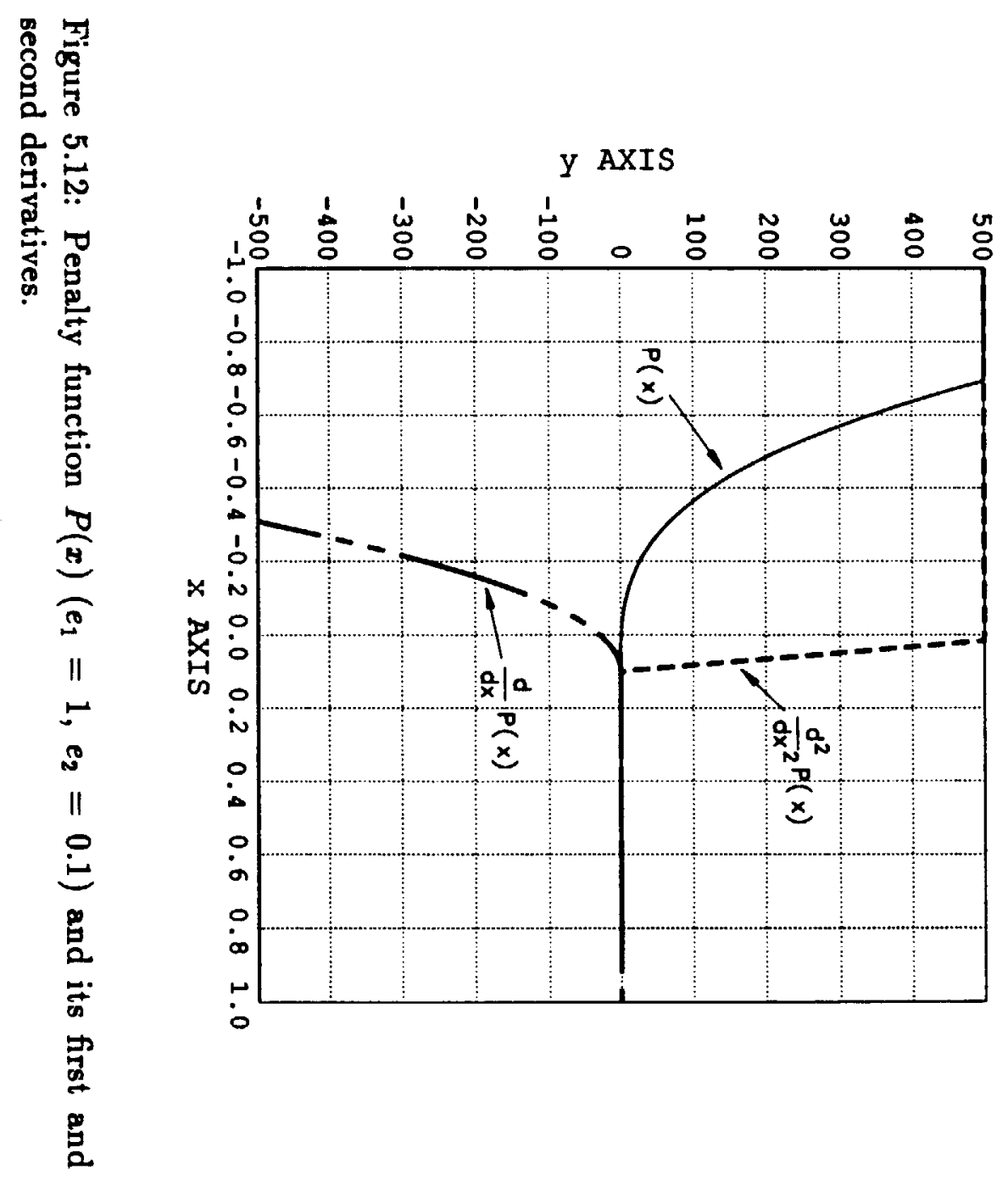


to be positive. When the cost function is being minimized and any of the values of $a^{2}, b^{2}$ or $R N$ is negative, the Penalty function will make the value of $\psi$ very large. The purpose of introducing the variable $R N$ is to assure that the center of the ellipse will be in the correct side of the cross-section curve, in our case, the interior of the cross-section curve. For example in Figure 5.8, the center of the ellipse is located outside the cross-section curve. For that case, the Penalty function $P(R N)$ will be a large positive number which will force the minimum of the cost function in (10) to a location where the center of the ellipse is in the interior of the cross-section curve. Recall that the results calculated for method 1 were obtained by means of the Steepest Descent technique. In method 3, a modified Newton-Raphson method is used. This method finds the minimum of the cost function by finding the zero of the gradient of $\psi$; however, in contrast to the usual Newton-Raphson algorithm, the modified version does not require a very good guess of the unknown parameters. After running some test cases it was found that the routine did not work very well with single precision on a VAX 8550 computer. After changing to double precision and adjusting the parameters $e_{1}$ and $e_{2}$, the routine was made to converge.

A careful study of the cost function was also conducted. By making some contour plots of $\psi$ and its gradient, we found that the minimum of $\psi$ as well as the zero of its gradient lie in a very steep sided valley where multiple zeros of $\nabla \psi$ exist. This is not a very desirable condition because it causes the search procedure to converge to a point where the cost function may not be the minimum. We are in the process of studying the possibility of modifying the cost function to avoid this difficulty. Another 
approach to avoid this difficulty is not to use a gradient-based method. These techniques, such as the simplex method, do not use the gradient of the cost function to find its minimum. The possibility of using these techniques will be studied in the future. 


\section{Chapter 6}

\section{Project Status}

The main accomplishments to date in the development of the HARP code are:

1. Specification and purchase of required Tektronix 4337 graphics workstation and associated software

2. Development of computer assisted graphics method of entering fuselage geometry via cross sections

3. Developed a modified periodic cubic spline to produce a smooth fit to the fuselage, with no anomalies

4. Developed routines to display the spline fit or continuous surface model of the helicopter

5. Conversion of the continuous surface model into a plate model for the low frequency MM analysis

6. Successful interface of the HARP code with the low frequency AMC method of moments analysis code 
7. Development of several possible procedures for constructing the high frequency ellipsoid UTD model of the fuselage from the continuous surface model.

8. First stages of development of the $\mathrm{X}$ windows menu driven user interface.

The main items proposed for the second year's effort are:

1. Develop an $\mathrm{X}$ windows menu driven user interface to permit the user to conveniently execute the HARP code.

2. Continue to develop methods, employing computer graphics, to allow the user to conveniently define the helicopter geometry. In particular, methods for defining the shape and location of wings and fins will be developed.

3. Improve the spline fit to the fuselage. In particular extend the present methods so that they can treat such special cases as an abrupt change in fuselage cross section. Also, obtain more information from the spline fit, such as surface normals and radii of curvature (needed to construct the UTD composite ellipsoid model of the fuselage).

4. Develop automated computer algorithms for obtaining the high frequency composite ellipsoid and flat plate model of the helicopter.

5. Continue to develop graphical displays of the helicopter geometry, including the continuous surface model, the MM plate model, and the UTD composite ellipsoid and flat plate model. 
6. Develop graphical methods for displaying the radiation patterns.

7. Improve the low frequency AMC or high frequency NEWAIR3 codes if necessary.

8. Expand the hardware capabilities and necessary software support to augment the project goals in the coming year (see Appendix D). 


\section{Bibliography}

[1] R.G. Kouyoumjian and P.H. Pathak, "A Uniform Geometrical Theory of Diffraction for an Edge in a Perfectly-Conducting Surface," Proc. IEEE, Vol. 62, pp. 1448-1461, Nov. 1974.

[2] P.H. Pathak, N. Wang, W.D. Burnside and R.G. Kouyoumjian, "A Uniform GTD Solution for the Radiation from Sources on a Convex Surface," IEEE Trans. on Antennas and Propagation, Vol. AP-29, No. 4, pp. 609-622, July 1981 .

[3] R.F. Harrington, "Field Computations by Moment Methods," MacMillan, New York, 1968.

[4] W.D. Burnside, J.J. Kim, B. Grandchamp, R.G. Rojas and P.H. Law, "Airborne Antenna Radiation Pattern Code User's Manual," OSUESL Technical Report 716199-4, generated under Grant NSG 1498, for NASA Langley Research Center, Sept. 1985.

[5] H. Späth, "Spline Algorithms for Curves and Surfaces", Utilitas Mathematica Pub., Inc., 1974.

[6] R. Bartels, J. Beatty, and B. Barsky, "An Introduction to Splines for Use in Computer Graphics and Geometric Modeling" , Morgan Kaufmann Pub. Inc., 1987.

[7] T. Lyche, and L. Schumaker, "Mathematical Methods in ComputerAided Geometric Design", Academic Press, 1989.

[8] C. De Boor, "A Practical Guide to Splines" , Springer-Verlag, New York, Inc., 1978.

[9] P. Prenter, "Splines and Variational Methods" ,Academic Press, 1975. 
[10] G. Farin, "Curves and Surfaces for Computer-aided Design: a Practical Guide",Academic Press, 1988.

[11] E.H. Newman, "An Overview of the MM/Green's Function Method in Electromagnetics," Proc. IEEE, Vol. 76, March 1988, pp. 270-282.

[12] W.H. Press; B.P. Flannery; S.A. Teukolsky; W.T. Vetterling; Numerical Recipes: The Art of Scientific Computing; Cambridge University Press, Cambridge; 1986.

[13] R.L. Burden; J.D. Faires; Numerical Analysis, 3rd.ed.; PWS-Kent Publishing Company, Boston; 1985.

[14] J.J. Dongarra; C.B. Moler; J.R. Bunch; G.W. Stewart; LINPACK Users' Guide; Society for Industrial and Applied Mathematics, Philadelphia; 1979.

[15] M. Athans, M. Dertouzos, R. Spann, S.J. Mason, Systems, Networks, and Computation, McGraw-Hill, New York, 1974.

[16] J.J. Kim, "Simulation and Analysis of Airborne Antenna Radiation Patterns," OSU-ESL Technical Report 716199-1, prepared under Grant No. NSG 1498 for NASA Langley Research Center, Dec. 1984.

[17] R.G. Rojas, Y.C. Chen, W.D. Burnside, "Improved Computer Simulation of the TCAS III Circular Array Mounted on an Aircraft," OSUESL Technical Report No. 716199-12, prepared under Grant NSG 1498 for NASA Langley Research Center, Mar. 1989.

[18] W.D. Burnside, N. Wang and E.L. Pelton, "Near Field Analysis of Airborne Antennas," IEEE Trans. on Antennas and Propagation, Vol. AP-28, pp. 318-327, May 1980.

[19] R.G. Rojas, P. Law and W.D. Burnside, "Simulation of an Enhanced TCAS III System in Operation," OSU-ESL Technical Report No. 716199-9, prepared under Grant NSG 1498 for NASA Langley Research Center, Oct. 1987.

[20] R.G. Hartenstein, "Analysis of Airborne Antenna Systems using Geometrical Theory of Diffraction and Moment Method Computer Codes," 
OSU-ESL Technical Report No. 716199-6, prepared under Grant NSG 1498 for NASA Langley Research Center, August 1985.

[21] R.J. Marhefka and W.D. Burnside, "Numerical Electromagnetic CodeBasic Scattering Code NEC-BSC (Version 2) Part I: User's Manual, OSU-ESL Technical Report 712242-14, prepared under Contract N00123-79-C-1469 for Naval Regional Contracting Office, december 1982.

[22] M. Peters and E.H. Newman, "A User's Manual for the Method of Moments Aircraft Modeling Code," Ohio State Univ. Research Foundation Report 716199-14, prepared under grant NSG 1498 between the Ohio State University Research Foundation and the National Aeronautics and Space Administration, Langley Research Center, September 1989.

[23] E.H. Newman “A User's Manual for the Electromagnetic Surface Patch Code: ESP Version IV," Ohio State University ElectroScience Lab Report 716199-11, generated under Grant NSG 1498 for NASA Langley Research Center, August 1988.

[24] J.H. Richmond, D.M. Pozar, and E.H. Newman, "Rigorous NearZone Field Expressions for Rectangular Sinusoidal Surface Monopole", IEEE Trans. Ant. and Prop., Vol. AP-26, pp. 509-510, May 1978.

[25] E.H. Newman and D.M. Pozar, "Electromagnetic Modelling of Composite Wire and Surface Geometries", IEEE Trans. Ant. and Prop., Vol. AP-26, pp. 784-789, Nov. 1978.

[26] E.H. Newman and D.M. Pozar, "Considerations for Efficient Wire/Surface Modeling", IEEE Trans. Ant. and Prop., Vol. AP-28, pp. 121-125, Jan. 1980.

[27] D.M. Pozar and E.H. Newman, "Analysis of a Monopole Mounted at or Near the Edge of a Wedge", IEEE Trans. Ant. and Prop., Vol. AP-29, pp. 488-495, May 1981.

[28] D.M. Pozar and E.H. Newman, "Analysis of a Monopole Mounted Near an Edge or a Vertex", IEEE Trans. Ant. and Prop., Vol. AP-30, pp. 401-408, May 1982. 
[29] E.H. Newman and P. Tulyathan, "A Surface Patch Model for Polygonal Plates", IEEE Trans. Ant. and Prop., Vol. AP-30, pp. 588-593, July 1982.

[30] E.H. Newman and M.R. Schrote, "On the Current Distribution for Open Surfaces", IEEE Trans. Ant. and Prop., Vol. AP-31, pp. 515518, May 1983.

[31] E.H. Newman, P. Alexandroupoulos, and E.K. Walton, "Polygonal Plate Modeling of Realistic Structures", IEEE Trans. Ant. and Prop., Vol. AP-32, pp. 742-747, July 1984.

[32] E.H. Newman, "The Equivalent Separation(s) for the Self-Impedance of Thin Strips", IEEE Trans. on Antennas and Propagation, Vol. AP. 35, pp. 110-113, Jan. 1987.

[33] E.H. Newman, "Generation of Wideband Data from the Method of Moments by Interpolating the Impedance Matrix", IEEE Trans. on Antennas and Propagation, Vol. AP-36, pp. 1820-1824, Dec. 1988.

[34] E.H. Newman and R.J. Marhefka, "Overview of MM and UTD Methods at The Ohio State University," Proc. of the IEEE, Vol. 77, pp. 700-708, May 1989. 


\section{Appendix A}

\section{High Frequency Techniques}

A computer code, written in standard Fortran 77, based on UTD solutions has been developed at The Ohio State University (OSU) to investigate the radiation patterns of antennas mounted on a fuselage which is modeled by a composite ellipsoid [4]. The Aircraft code [4] was originally developed by Prof. Burnside and the present code (NEWAIR3) is the third version. This computer code is used to compute the near and far zone radiated fields for antennas mounted on a composite ellipsoid and in the presence of a set of flat plates. Since the NEWAIR3 code is based on the uniform geometrical theory of diffraction (UTD), the structures that can be analyzed have to be electrically large. In terms of the scattering from plate structures, this means that each plate should have edges at least a wavelength long. In terms of the composite ellipsoid structure, its major and minor radii should be at least a wavelength in extent. In addition, each antenna element should be at least a wavelength from all edges. In some cases, the wavelength limit can be reduced to a quarter of wavelength for engineering purposes. This code has been used at OSU to solve a wide variety of 
antenna radiation problems $[17,18,19,20]$. In addition, it has been widely distributed and used by the aerospace industry.

Although the code was written specifically to analyze general aircraft structures, it is enough that it can allow the user to simulate a wide variety of complex electromagnetic radiation problems using the ellipsoid/plates model. For example, the composite ellipsoid can be used to accurately simulate the fuselage of an aircraft; whereas, the plates are used to represent the wings, stabilizers, stores, engines, etc. This code can also be used to simulate the radiation of an antenna mounted directly on a ship mast. In this case, the mast can be modeled by the composite ellipsoid with the other ship structures simulated by the flat plates. Note that the plates can be attached to the composite ellipsoid and/or to other plates. In fact, the plates can be connected together to form a box.

This code can also be used to model a helicopter when the radiating antenna is mounted on a curved surface. The helicopter can then be modeled by the composite ellipsoid and a set of flat plates. It is important to mention that the NEWAIR3 code can be used in conjunction with another code also developed at OSU and known as the Basic Scattering Code (BSC) [21]. In the BSC, the antenna cannot be mounted on a curved surface, but it can be placed on a flat surface or in free space. Thus, these two codes complement each other as shown in [20], where the NEWAIR3 and the BSC codes are used to solve a number of radiation problems.

The NEWAIR3 code has the flexibility to handle arbitrary pattern cuts in the near or far field regions. All the components of the radiated electric field are computed but only the $E_{\phi}$ and $E_{\theta}$ fields are stored in a 
binary file for later use; however, the code can be easily modified such that the user can store additional field components. Note that an arbitrary antenna type can be analyzed provided the current distribution across the aperture is known. This is done by approximating the distribution by a set of magnetic current elements mounted on the surface, or by electric current elements normal to the composite ellipsoid surface. The magnetic current elements have a cosine distribution along the magnetic current direction and a uniform distribution in the orthogonal direction. The normal electric current represents a monopole and its length can not be greater than a quarter wavelength. In the case of an antenna array, where mutual coupling effects between the array elements are important, an intermediate calculation is necessary before the NEWAIR3 code can be used. Since the NEWAIR3 code does not take into account the coupling between array elements, it is necessary to first calculate the mutual coupling effects which can be accomplished with a moment method code known as the Electromagnetic Surface Patch Code (ESP) [23] also available at The Ohio State University ElectroScience Laboratory. Once the induced currents are obtained by means of a moment method analysis, they can then be taken to be part of the input data to the NEWAIR3 code.

The way the NEWAIR3 code is usually used is as follows. First, a detailed drawing of the structure (with all the dimensions) is required. Once this is obtained, it is necessary to know the frequency of operation, the antenna locations and the type of antennas. Keeping in mind that the computer model has to accurately model the structure in the vicinity of the radiating antenna, a computer model is generated using the composite 
ellipsoid and flat plates. This means that many features of the structure located far (in terms of wavelengths) from the radiating antenna can be neglected or do not need to be modeled accurately. The next important step is to generate a model of the radiating antennas using the monopoles and slots mentioned above. As shown in $[18,19]$, where a circular array of top loaded monopoles is simulated, a wide variety of antennas can be modeled with the help of the ESP code [23]. Once the antenna models are obtained, the radiation patterns in the near and/or far field can then be obtained. Note that the present code will run a pattern cut of 360 points for a commercial aircraft model with one antenna element in approximately 1 minute on our VAX 8550 computer. 


\section{Appendix B}

\section{Low Frequency Techniques}

Low frequency or method of moments (MM) [3] techniques can be used to accurately and efficiently analyze the radiation from helicopter antennas when the maximum dimension of the aircraft is on the order of a few wavelengths or less. This section will provide a brief overview of the MM, and its use in computing the radiation pattern for antennas on helicopters.

The MM solution for the radiation from an antenna in the vicinity of a perfectly conducting body, such as a helicopter, proceeds in two basic steps. The first step is to obtain an integral equation for the current distribution on the antenna and on the conducting surface. This integral equation is exact, and is an expression of the boundary condition of zero tangential electric field field on the surface of the antenna and the conducting body. The next step is to solve the integral equation using the MM. The current is expanded in terms of $N$ basis or expansion functions, and $N$ weighted averages of the integral equation are enforced. This transforms the integral equation into an order $N$ matrix equation which can be solved for the $N$ coefficients in the original expansion for the current. Once the current is 
known, most parameters of engineering interest, such as input impedance, mutual coupling, or radiated fields (co and/or cross polarizations), can be found in a straight-forward manner.

The main advantage of MM solutions is high accuracy. The $M M$ is a direct numerical solution of the exact governing integral equation for the current distribution. All phenomenon of the problem, including surface waves, creeping waves, shadowing effects, etc., are automatically included in the MM solution. As $N$, the number of expansion modes increases, the MM solution can approach the exact result. A second advantage of MM solutions is that they can in practice be applied to the analysis of very complex or realistic shapes. The main limitation of MM solutions is a result of the fact that the number of unknowns in the MM solution is proportional to the electrical size of the body. For a conducting surface typically $N=40$ unknowns per square wavelength are required. On thin wires, typically 5 unknowns per wavelength are required. The computer storage requirements for $\mathrm{MM}$ solutions is proportional to $N^{2}$; while, the CPU time can be proportional to $N^{2}$ or $N^{3}$. As the frequency is increased, $N$ must be increased, and at some point the required computer CPU time and storage become impractical. MM solutions are most applicable when the surface area is less than about $10 \lambda^{2}$.

The "Electromagnetic Surface Patch" or ESP code is a user-oriented computer code, based upon the MM, for analyzing the radiation or scattering from a general interconnection of thin wires and polygonal plates [23]. The theoretical basis of the ESP code is described in several journal articles [24]-[34]. The ESP code is capable of analyzing an almost arbitrary thin 
wire antenna in the presence of an almost arbitrary perfectly conducting surface. A conducting surface, such as a helicopter, a ship, an automobile, etc., is modeled as an interconnection of several polygonal plates. The thin wires are used to model a wire antenna which may be radiating in the presence of the conducting surface.

A special purpose version of the ESP code has recently been generated for the purpose of analyzing the radiation from antennas on helicopters. The code is referred to as the "Aircraft Modeling Code" (AMC) [22]. Basically the AMC provides the user a reasonably simple and straight forward procedure for defining the helicopter geometry. The helicopter fuselage is specified by defining its cross section at several stations from its nose to its tail. At each station the cross section is defined in terms of a polygon which approximates the actual cross section shape. AMC takes this cross section information and generates polygonal plates which approximately model the fuselage shape. The accuracy of the model increases as the number of cross sections increases. The user can also specify various wings, fins, rotor blades, etc., which are attached to the fuselage, as additional polygonal plates. Finally, a few wire segments are used to model a transmitting antenna mounted on the fuselage or on the wings. Once the polygonal plates and wires are defined, the radiation from the antenna can be analyzed as in the ESP code.

The insert in Figure B.1 shows a helicopter model generated by AMC. The fuselage cross sections are modeled by eight sided polygons. The cross sections are interconnected to form a polygonal plate model of the fuselage. Attached to the fuselage are other plates representing wings or fins. Finally, 
a short dipole is located on the underside of the fuselage. Figure B.1 shows a comparison of the computed (dashed line) and measured (solid line) far zone radiation pattern for the dipole in the presence of the helicopter. 

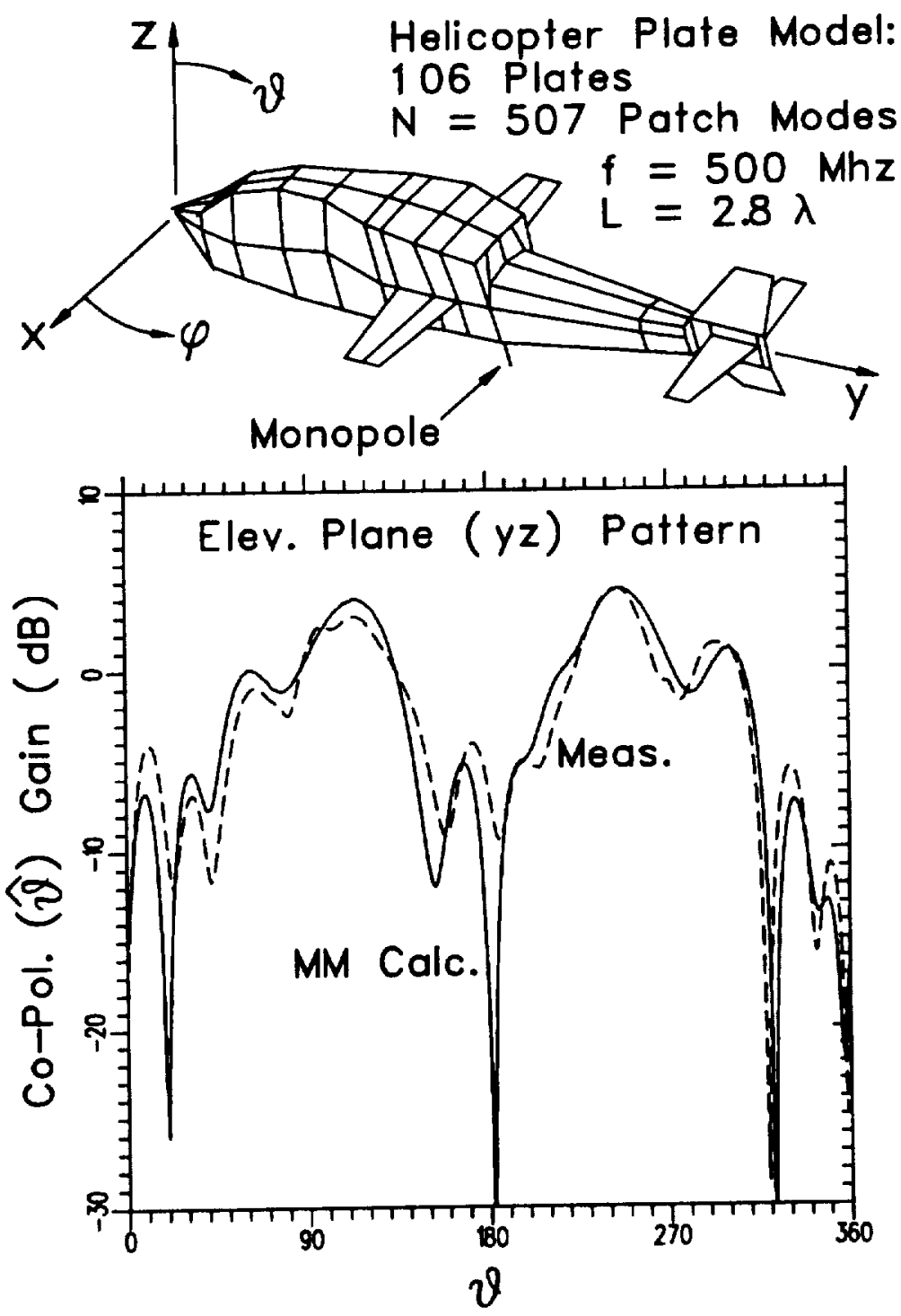

Figure B.1: The computed (dashed line) and measured (solid line) far zone pattern of a short monopole on the underside of a helicopter computed by AMC. 


\section{Appendix $\mathbf{C}$}

\section{The Tektronix 4337 Workstation}

This appendix will describe the hardware platform being used to develop the HARP code. The Tektronix 4337 workstation provides a consistent Unix operating system with a complete implementation of the $\mathrm{X}$ Window System, while providing simultaneous 3-D graphics display capabilities.

The workstation platform is a true stand-alone system, complete with sufficient memory, hard disk storage and peripheral devices (including a color wax printer) to completely perform all the necessary steps to implement the HARP code package. The Motorola 68020 processor is supported by 12 megabyte of physical memory, and is configured with 1.2 gigabyte of hard disk storage. One of the singular features of this workstation is its ability to support both an $\mathrm{X}$ Window System (version 11) and true 3-D graphics, complete with hardware rotation, zoom and pan. This allows the operater the convenience of a menu driven windowed interface coupled with high speed $(350,000$ 3-D vectors/sec.) color graphic representation of the helicopter. 
The Tektronix 4337 is capable of performing not only the user interface portion of the HARP code, but is also well suited to tackle the necessary electromagnetic computations for a variety of cases. For more demanding requirements, the 4337 can readily be field upgraded to a Tektronix XD88 Superworkstation, thereby providing a six-fold increase in raw compute power, while retaining the outstanding graphics performance. In either configuration, the workstation provides complete network compatability to existing systems via ethernet protocols, thereby allowing users to access the shared compute resources of available networks. 


\section{Appendix D}

\section{The Tektronix Hardware/Software Improvements}

During the second year's efforts, we will be expanding the capabilities of the system through hardware and associated software improvements. These improvements include:

1. Tektronix 4200F4D Valuator Dials

2. Tektronix 41P42 Teknicad Software

3. Tektronix 43P71-1F Software Terminal Interface (STI)

4. Tektronix 41P70-1H Graphics Kernal System (GKS)

5. Tektronix 4300P37 Fortran 77 Compiler

The first three items listed are peripheral devices which will provide enhanced input and output from the system for the operator. The remaining four items are software items which are in support of the overall project effort. 
722792-1, “The Helicopter Antenna Radiation Prediction Code”, Klevenow, Lynch, Newman, Rojas, Scheick, Shamansky, Sze, August 1990 